\title{
A heuristic model of sensory adaptation
}

\author{
Donald H. McBurney and Carey D. Balaban \\ University of Pittsburgh, Pittsburgh, Pennsylvania
}

\begin{abstract}
Adaptation is a universal process in organisms as diverse as bacteria and humans, and across the various senses. This article proposes a simple, heuristic, mathematical model containing tonic and phasic processes. The model demonstrates properties not commonly associated with adaptation, such as increased sensitivity to changes, range shifting, and phase lead. Changes in only four parameters permit the model to predict empirical psychophysical data from different senses. The relatively prolonged time courses of responses to oral and topical capsaicin are used to illustrate and validate this mathematical modeling approach for different stimulus profiles. Other examples of phenomena elucidated by this modeling approach include the time courses of taste sensation, brightness perception, loudness perception, cross-adaptation to oral irritants, and cutaneous mechanoreception. It also predicts such apparently unrelated phenomena as perceived alcohol intoxication, habituation, and drug tolerance. Because the integration of phasic and tonic components is a conservative, highly efficacious solution to a ubiquitous biological challenge, sensory adaptation is seen as an evolutionary adaptation, and as a prominent feature of Mother Nature's small bag of tricks.
\end{abstract}

"Mother Nature's bag of tricks is small."

—Gregory Kimble, 1990

The purpose of this article is to review the properties of sensory adaptation and to suggest a heuristic model that organizes the many phenomena it subsumes. We will further propose that adaptation is one of the most fundamental behavioral principles and, as such, can serve to unify many apparently unrelated behavioral phenomena.

The term adaptation is used so widely, and has been studied so extensively, that it may surprise some readers that there is considerable confusion as to what the term means. Probably the most typical use of the term is to refer to a decline in the response of a sensory system $^{1}$ to a steady stimulus. Thus, Partridge and Partridge (1993) said, "adaptation occurs when a constant stimulus causes an initial large response that dies away to a lower maintained response" (p. 80). The definition of adaptation as a time-dependent decrement in response magnitude has created a general perception that, as a corollary, adaptation reflects a decline in sensitivity of a receptor, and hence, a net loss of information (see, e.g., Dworkin, 1993; Loewenstein, 1971). As long ago as 1929, however, Bekesy (1960) demonstrated that adaptation can increase the sensitivity of a receptor to changes in an auditory stimulus. And more than four decades ago, an article was published with the title "Adaptation: Loss or gain of sensory information?" (Keidel, Keidel, \& Wigand, 1961) that extended Bekesy's work.

Adaptive phenomena have been documented at levels from sensory receptors to sensations expressed by the whole organism. Sensory adaptation at the level of recep- tors is universal across modalities, with relatively short time courses (order of milliseconds or seconds). Psychophysical adaptation also occurs in these senses, but the time course is prolonged. However, the common features (or templates) of adaptive responses are sufficiently similar that they can be understood within a common heuristic framework.

One indication of the attention given to adaptation may be seen in the treatment given the topic over the years in leading textbooks and handbooks that deal with the senses. In 1951, Stevens's Handbook of Experimental Psychology had 11 references to adaptation. Although the 2002 edition (Pashler, 2002) had been expanded to four volumes, the term adaptation did not appear once. Nor does Goldstein's leading textbook, Sensation and Perception (7th ed., 2007) include the term in its index. Goldstein (personal communication, 2007) has said that

adaptation may be treated as a phenomenon in its own right (i.e., what are the facts, mechanisms governing visual adaptation, taste adaptation, etc.) or as a tool which can be used to elucidate underlying mechanisms (i.e., the selective adaptation literature which exists in vision, taste, and tactile senses). Many authors have tended to treat adaptation as a tool rather than as a phenomenon.

Several concurrent trends have contributed to the loss of interest in unifying features of adaptation across the senses. One major trend has been the fragmentation and subspecialization of expertise within traditional disciplines of psychology, physiology, and neuroscience, to the extent that scientists who study the different senses

C.D. Balaban, cbalaban@pitt.edu 
form virtually different scientific communities. These disciplinary communities are often characterized as silos or stovepipes, because the narrow focus inhibits the generalization of unifying concepts across community boundaries. A second major trend has resulted from the explosion of new information in cellular and molecular biology and the associated focus on explaining biological phenomena at the level of the genome, proteome, metabolome, and lipidome. A third related trend is the reorientation of training toward "skills for success" within a particular discipline. Exposure to the classical approach that there are unifying concepts such as adaptation has been a casualty. This consequence of Aristotelian reductionism is not new; it was noted nearly a millennium ago by Yehuda Halevi, who said, "Let not the wisdom of the Greeks beguile you; which has no fruit, only flowers." Contemporary research has focused on "flowers," interpreting findings within the narrow context of a particular sense and mechanism. The "fruits" are general principles, heuristics, or mathematical models that generalize across these specific cases.

This article uses a simple heuristic mathematical model for sensory processing to illustrate that many aspects of phenomena defined as habituation and adaptation are predicted by the dynamic characteristics of information processing. The discussion proceeds in four stages:

1. The definitions of adaptive phenomena are problematic, with conflicting, arbitrary definitions of adaptation, habituation, stimulus failure, and fatigue. As a result, we focus on a "black box" analysis of the properties of the response rather than on issues arising from conflicting terminologies.

2. Since sensory afferent fibers carry both phasic (rapidly adapting) and tonic (slowly adapting) signals, we present a simple additive model of these signals that reproduces both dynamic response characteristics and adaptation. Among other properties, the model indicates that adaptation can be viewed as trading off information about absolute stimulus magnitude in favor of sensitivity to changes in stimulus intensity. From this perspective, adaptation represents both a gain and a loss of information.

3. Explanatory capabilities for existing psychophysical data.

4. Implications for other behavioral processes.

\section{The Problem of Distinguishing Adaptation From Other Processes}

Although Partridge and Partridge's (1993) definition of adaptation is representative, it has been common to distinguish adaptation from other similar processes on a number of bases. Thus, adaptation has been distinguished from stimulus failure if the decrease in response is the result of a purely mechanical failure of a steady stimulus to continue affecting the receptor, as when the skin ceases to continue to be deformed by a steady touch. It has been distinguished from fatigue on the basis that the receptor is still capable of responding if the stimulus is changed, whereas in fatigue it would not be. It has been distinguished from habituation on the basis that the latter has a longer time course and involves central and/or associative mechanisms. It has been distinguished from accommodation on the basis that the latter is an increase in a neuron's threshold when a stimulus is increased slowly.

At this point, we simply note that these distinctions are all problematic and have resulted in much confusion. This confusion is magnified by the lack of consistent application of these definitions to investigation of different sensory modalities. For example, the term fatigue is used widely in the study of audition to refer to the same phenomena that are called adaptation in most other senses. It is also worth noting that the term adaptation is used to refer to changes in motor performance of vestibuloocular reflexes in response to experimental manipulations of visual information impinging on the retina (e.g., the body is rotated in a lighted room with magnifying lenses, minifying lenses, or reversing prisms in front of the eyes). By contrast, the term habituation is used if there is simply a decrease in the magnitude of vestibulo-ocular reflexes after repeated stimulation.

This is far from a novel insight. Geldard (1968) summed up a discussion of these issues as follows.

Sufficient frustration over conflicting meanings of the oft-times competing terms, adaptation, fatigue, habituation, and equilibration, has been expressed during [this symposium] to prompt the question whether it is not time to organize some resistance to the tyranny of these words. Our annoyance with this quartet of terms springs from the devious historical roots of several allied sciences. Each word could be traced to many sources; each could be shown to have become immersed in ambiguity. (p. 593)

\section{A Definition of Adaptation}

In the broadest sense of the term, adaptation is defined by the various implications of the fact that sensory systems have time-varying properties. Hensel (1968) came close to our definition of adaptation:

The general meaning of the term adaptation is nothing more than the fact that a time factor is involved in the correlation of the external stimulus and the neural output, let us say, of a receptor. The whole system is sort of a black box with a time factor built in. If you have a rectangular stimulus, then the neural discharge frequency is high at first and gradually diminishes. In a general sense, this is adaptation. (p. 328)

Foster and Smyth (1980) captured this idea and noted its significance, as follows.

A fundamental property of sensory systems is that when an instantaneous stimulus (a pulse) is given, the response is not instantaneous, but is spread out in time. ... This property is ... essential to the biological usefulness of any sensory system. (p. 612)

Despite the traditional reliance on the faster time course of a response decrement as the defining criterion for distinguishing sensory adaptation from habituation, it is critical to note that this distinction cannot be applied consistently. A key example is provided by adaptation to 
capsaicin, the active ingredient in chili peppers that produces a burning sensation. Karrer and Bartoshuk (1991) and McBurney, Balaban, Christopher, and Harvey (1997) found that adaptation to capsaicin reduced the burning sensation over days; Karrer and Bartoshuk reported a decrement lasting up to 6 days after a single exposure. A second example is provided by the olfactory system. According to an ancient proverb, stercus cuique suum benet olet, or "everyone's dunghill smells all right to himself" (Critchley, 1986, p. 5). Dalton and Wysocki (1996) found that the threshold for detection of an odor increased more than 16-fold when subjects were exposed chronically to the odorant over the course of 2 weeks. A third example appears in the literature regarding temperature sensitivity. Repeated exposure to cold over several days lowers felt discomfort (Brück, Baum, \& Schwennicke, 1976). Thus, the time course alone is a poor basis for distinguishing adaptation from other processes.

The focus of this article is on the response to a stimulus that varies only in intensity over time. We do not address phenomena such as masking, the McCullough effect, or other results of perceptual rearrangement, which may involve different stimuli and/or associative learning.

\section{A Black Box Approach to Adaptation}

The inconsistent nomenclature for adaptation appears to be an unfortunate consequence of a tendency to focus on the adaptation of each sensory system as an independent process, rather than as a special case of a general set of principles or processes of all sensory systems. Furthermore, the development of a unified approach to adaptation faces the challenge of explaining phenomena, not only across organisms and sensory systems, but also at different levels of organization within a single organism and sensory system. For example, adaptation in the visual system occurs within multiple cell populations in different structures. Within the retina alone, adaptation has been documented within the photoreceptors, the bipolar cells, and the amacrine cells, which work in a hierarchical manner, with feedback among levels (Shapley \& EnrothCugell, 1984). Furthermore, several forms of "molecular" adaptation have been identified. As discussed by Koshland (1980), destruction and generation of receptors is used in various biological systems as a way of controlling sensitivity. He noted that exposure to hormones causes their receptors to internalize so that they are no longer exposed to the circulating hormone. Furthermore, in bacteria there is a proportionality between the number of receptors and the ambient stimulus intensity.

An additional form of adaptation at the molecular level has been described for the expression of the rod photopigment rhodopsin. Penn and Williams (1986) reported that the amount of rhodopsin varied with the level of ambient illumination, such that the daily photon catch is invariant in rats. This phenomenon, termed photostasis, was suggested as an adaptation of the animal to differing levels of ambient illumination throughout the year.

Despite the apparent complexity of these adaptive phenomena at multiple levels within organisms, the defining behavioral profile of adaptation is remarkably simple and consistent across organisms and sensory systems. Adrian (1928) recognized that the response patterns of nerve fibers to sensory stimuli resembled a summation of an exponential rapidly adapting process with a slowly adapting response component. Similar temporal response characteristics are shown by other sensory afferents, such as retinal ganglion cells (Rodieck, 1973), auditory nerve fibers (Kiang, 1966), and vestibular nerve fibers (Wilson \& Melvill Jones, 1979). A rapidly adapting response is a form of change detector; it is sensitive to changes in stimulus magnitude. It can also be described as a high-pass filter because it is sensitive to high-frequency but not low-frequency stimulus variations. A slowly adapting response is a form of level detector. It is insensitive to rapid stimulus fluctuations, but detects steady (or tonic) stimulus intensity. It can also be described as a low-pass filter, because it is insensitive to high-frequency information.

The behavioral relevance of level detection and change detection is well known in the various senses. It is, therefore, reasonable to view the behavioral (or psychophysical) phenomena as reflections of underlying processes, with similar high-pass and low-pass filtering characteristics. This line of thought predicts that adaptive phenomena can be explained by a formal model incorporating a summation of parallel rapidly adapting (phasic) and slowly adapting (tonic) components. Each sensory system utilizes a different weighting of these components to reflect factors such as the biological differences in sensory transduction processes and the relative importance of detecting absolute versus relative stimulus intensities.

Modeling and simulation methods are one powerful way of demonstrating rigorously that a concept generalizes across sensory domains. Advances in computational capabilities and simulation methods provide a rich array of tools for testing the ability of explicit constructs to explain phenomena in different sensory modalities. These tools let investigators appreciate that even relatively simple, biologically plausible models are sufficient to display rich and complex behaviors that would otherwise appear to be phenomenologically (and, therefore, mechanistically) distinct from one another. This article will illustrate how a simple dynamic model of sensory processing can both account for individual differences in response patterns and generate phenomena that have been termed sensitization, tachyphylaxis, fatigue, habituation, desensitization, cross-sensitization, and cross-desensitization. This model is intended to elucidate temporal properties of sensations in response to simple stimuli. It is not intended for phenomena such as prism adaptation and responses to spatiotemporal stimulus variations.

A prototype of a mathematical model that can predict adaptation and habituation is illustrated schematically in Figure 1A. Component responses are shown schematically in Figure 1B. The frequency-response characteristics of models with a small tonic component (thick line) and relatively large tonic component (dashed line) are shown in a Bode plot in Figure 1C. Similar frequency domain profiles from the literature for temporal modulation in vision (Figure 7), taste (Figure 8), hearing (Figure 9), and 
A

Model

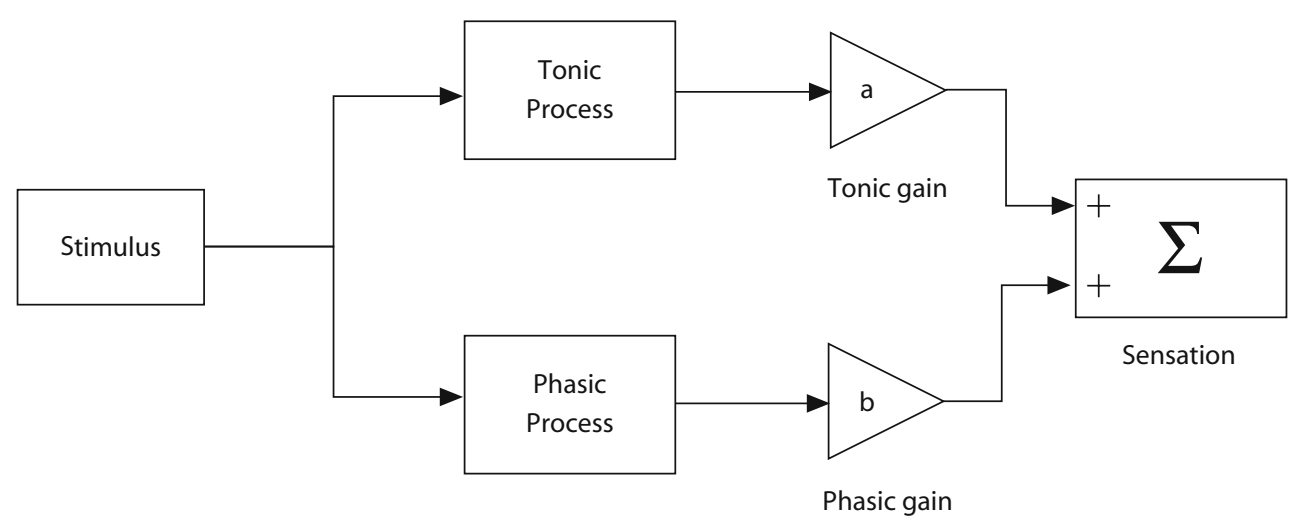

B

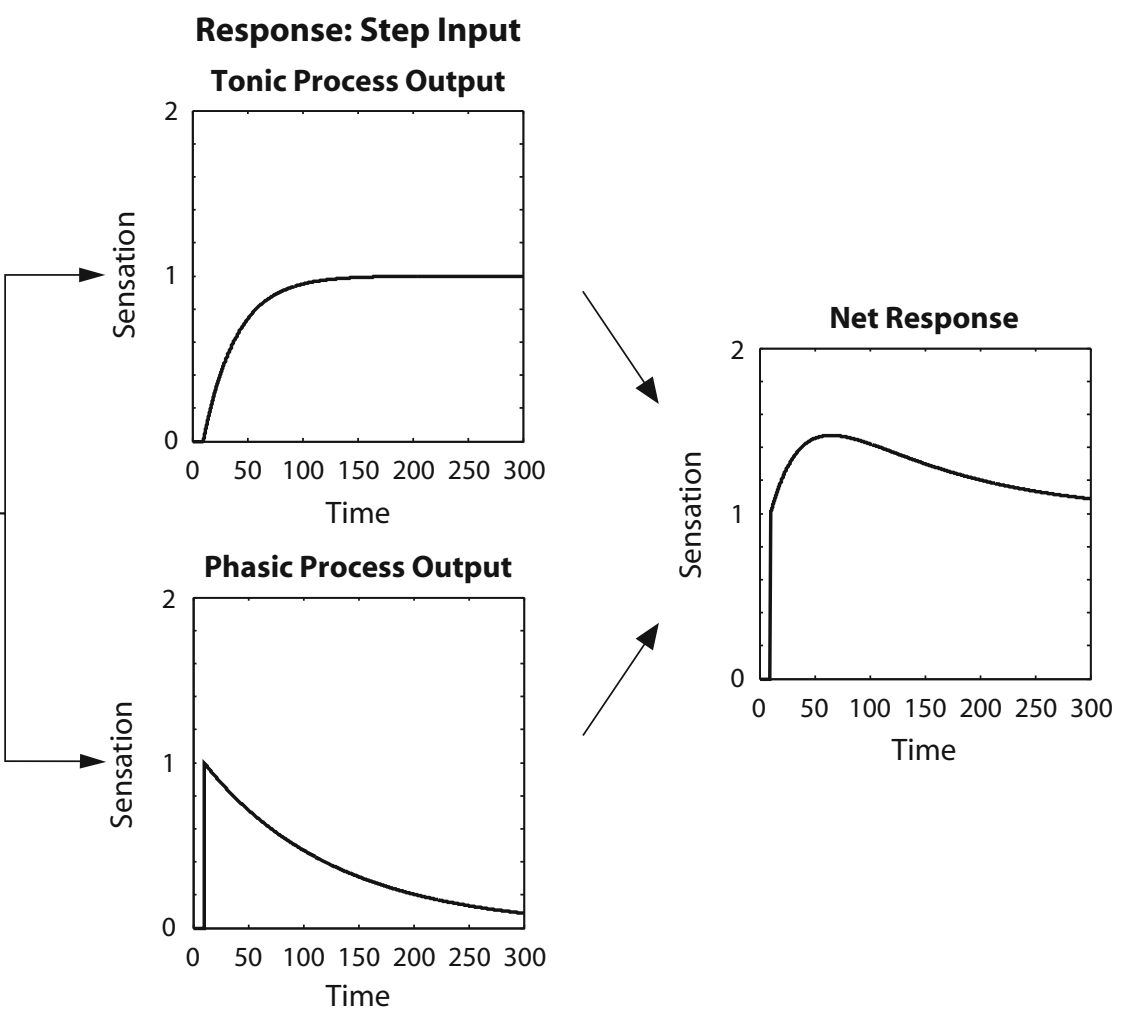

Figure 1. Heuristic phasic-tonic model for adaptation. (A) Block diagram of the model structure. (B) The response of each component of the model to a step function input. The components are described in detail in the text. (Continued on next page)

touch (Figure 10) are discussed in a later section of this communication.

The model consists of two basic components: a tonic response component and a phasic response component. The tonic component is a first-order low-pass filter and the phasic component is a second-order band-pass filter, which can be modeled in many cases as a first-order high-pass filter that detects rapid changes in the magnitude of the input from the tonic component. The outputs of the phasic and tonic components are added at a central site to produce the sensation. The relative magnitude of the tonic and phasic components determines the shape of the frequency-dependent dynamic response characteristics (Figure 1C).

These simple linear systems were simulated in MATLAB (The MathWorks), a standard engineering software package, using a first-order, low-pass filter for the tonic process and a first-order, high-pass filter for the phasic process. The basic components of this model are all physiologically plausible properties of sensory systems. Two temporal components of sensory afferent information are represented in the general model by independent phasic and tonic signal components. Since sensory modalities differ in their responsiveness to absolute stimulus 
C Bode Plot
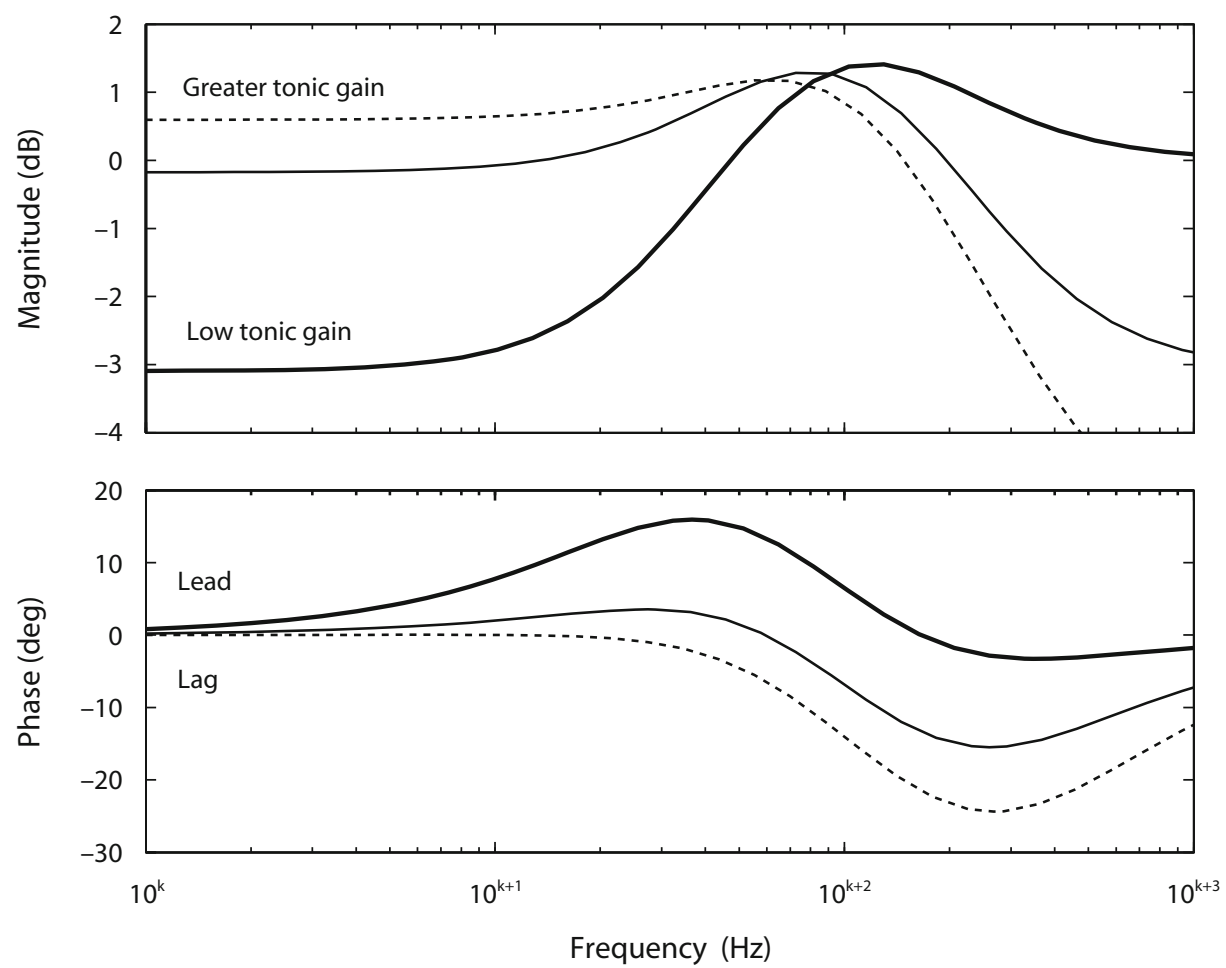

Figure 1 (Continued). (C) This Bode plot shows examples of a range of shapes of frequencyresponse characteristics produced by different relative contributions of tonic and phasic components. When the tonic response gain is relatively small (thick line), the response is dominated by the phasic process (band-pass high gain and phase-lead region). As the relative magnitude of the tonic gain is increased (thin and dashed lines), the response increasingly assumes a low-pass filter configuration.

magnitude versus changes in stimulus magnitude, the relative contributions (or weightings) of these components will vary with the sensory modality. For example, in the case of gustation, the system is predominantly phasic. By contrast, the tonic component is more dominant in the auditory system. Finally, the central processing component reflects the temporal characteristics of processing of the afferent input and response generation. The properties of the model in Figure 1 can be easily appreciated by considering the behavior of the two dynamic components, the tonic and phasic processes.

The sections that follow illustrate that this model, based on simple interactions between phasic and tonic sensory signals, is sufficient to generate a variety of properties associated with adaptation in different sensory systems. Furthermore, these basic properties are not altered when the phasic component becomes a second-order system by receiving its input from the tonic component. Although the values of these parameters will vary with the sensory modality, the fundamental structure of the model provides a unifying framework for approaching a broad range of phenomena termed adaptation and habituation.

The temporal responses of the phasic and tonic components to a staircase of increasing stimulus intensity steps are contrasted in Figure 2. The upper panels of Figures 2A and $2 \mathrm{~B}$ each show a series of increasing step stimuli. The middle panels show the responses of a phasic (Figure 2A) or tonic (Figure 2B) component with a time constant that is short with respect to the duration of a step. The lower panels show responses when the time constant is of long duration with respect to the duration of a step. These responses illustrate the basic properties of these components.

\section{Change Detection: \\ Properties of the Phasic Component}

1. When the time constant is short with respect to the duration of each step, the individual responses of the phasic component adapt/habituate (Figure 2A, middle panel). This property results in a series of equal-magnitude responses that reflect the change in the stimulus intensity and that decline exponentially to zero. This exponential decline in the phasic response is commonly cited as the defining criterion for adaptation.

2. When the time constant is long with respect to the duration of each step, the phasic responses show temporal summation (Figure 2A, lower panel). The maximum response amplitude (re: baseline) occurs at an infinitesimally small interval between steps, when it approaches the response to a single step of magnitude $2 \mathrm{~S}$. The peak response then declines exponentially with increasing in- 
A

Step Response of Phasic Component
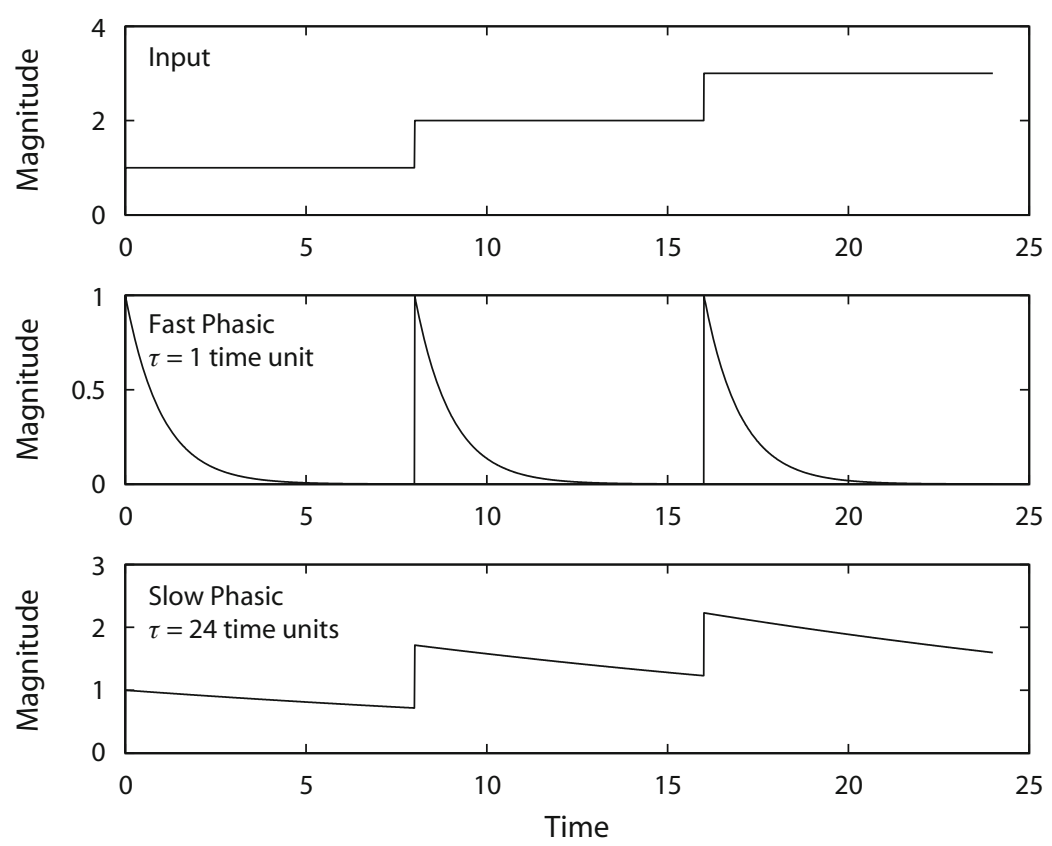

B Step Response of Tonic Component
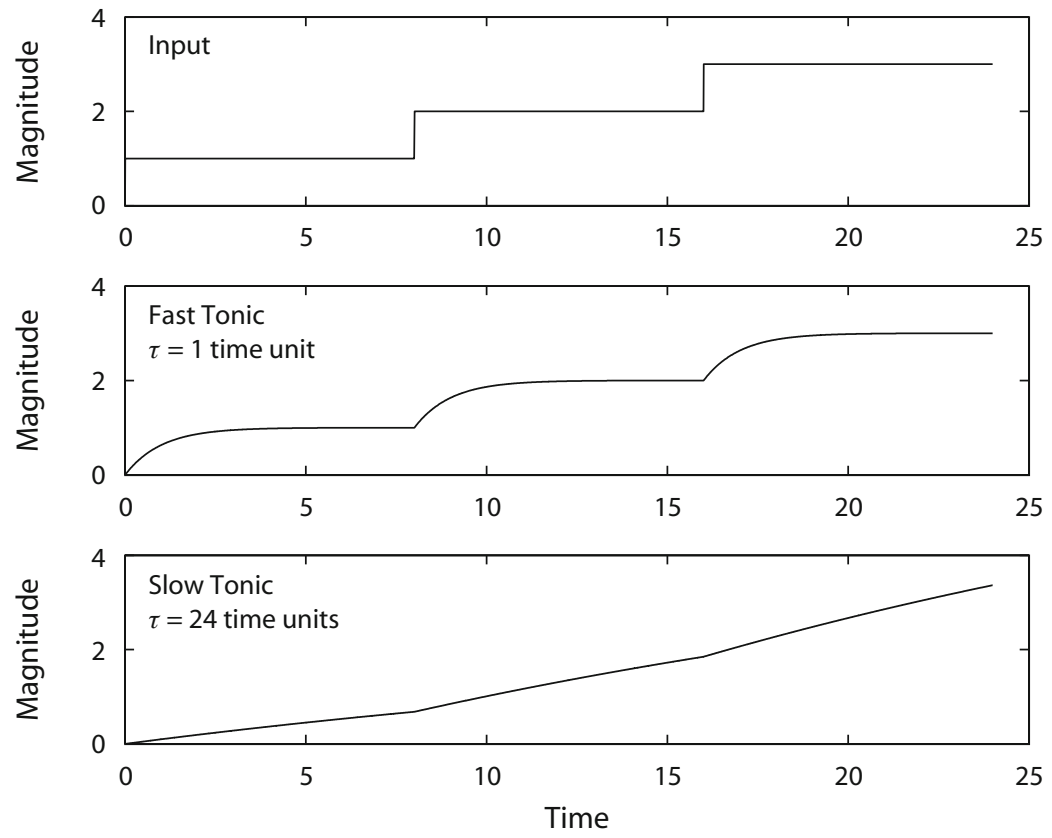

Figure 2. (A) Response of a phasic system to a series of step inputs. The middle panel shows the response of a phasic system with a short time constant. Note that it adapts completely prior to the next step. The lower panel shows the response of a phasic system with a long time constant. Because the adaptation is slow, the responses partially summate. (B) Responses of a tonic system to the same step inputs. A tonic system does not adapt. However, a tonic system with a short time constant (middle panel) reaches a plateau prior to the next step input. The slow tonic response does not reach a plateau prior to the next stimulus. 
tervals between the steps, finally reaching a minimum value when the interval is sufficient to produce complete adaptation to the first response (an interval of approximately three time constants).

3. Now, let us consider steps that change in magnitude. If changes in stimulus magnitude occur at intervals greater than three time constants, the responses are insensitive to a preadapted baseline intensity. This property is true for the entire time course of the response, including the peak response, and for all intensities. As a result, the phasic component is sufficient to produce range shifting of responses, such that the response reflects the difference between the stimulus and the adapted level rather than the absolute stimulus intensity.

4. A phasic system responds to change (i.e., first derivative) of a stimulus. The maximum change in a stimulus occurs before the stimulus reaches its maximum. Hence, the response is greatest during a period of maximum change in the input, which precedes the maximum input. Since this feature is true for any stimulus profile, the result of a phasic processor is an output that leads the input in time (Pringle \& Wilson, 1952). As noted by Partridge and Glaser (1960), this phase lead is a quintessential property of a phasic input, which functions as a "rate-of-change detector." The prediction that the peak response occurs prior to the peak input magnitude may be the most counterintuitive property of a phasic process.

\section{Level Detection:}

\section{Properties of the Tonic Component}

1 . The magnitude of the response of a tonic system to an escalating series of sustained steps is insensitive to the duration of each step. When the duration of each step is short with respect to the time constant, the responses fuse into a single rising response (Figure 2B, lower panel). Conversely, a series of step responses ensues when the duration of each step is short with respect to the time constant (Figure 2B, middle panel); hence, a tonic system does not show "adaptation." Since the peak response of a tonic system is strictly a function of absolute stimulus magnitude, it does not show range shifting.

2 . The response of a tonic system peaks after the maximum magnitude of a sensory input. For a sinusoid, the tonic system will lag the stimulus.

\section{Summation of Phasic and Tonic Components: Linear Superposition}

1. An important property of linear systems is that the sum of the outputs of two linear systems is itself linear. As a result, phasic and tonic components can be analyzed independently, and the sum of the component responses must equal the output of the total system. For sinusoidal inputs, this property implies that both the magnitude and phase of the output are determined by the relative influence of phasic and tonic components.

2. The responses to time-varying stimuli are determined by the temporal filtering characteristics of phasic and tonic systems. A standard approach to understanding filtering properties of these linear systems is to examine the transfer function, which displays the effects of the system on the magnitude and the phase (timing) of the response as a function of the input frequency. For a linear system, this transfer function is sufficient to predict responses to any arbitrary, time-varying stimulus (e.g., ramps and impulses).

3. Sensory processing involves a combination of tonic and phasic components, varying from systems that are primarily phasic to systems that are primarily tonic. In addition to predicting the time course of responses to individual stimuli, the relative contributions of these components also determine the maximum responses to stimuli of given intensities under varying adaptation states. A phasic system shows range shifting (Figures $3 \mathrm{~A}$ and $3 \mathrm{C}$ ), whereas a tonic system does not adapt (Figures 3B and 3D). The addition of parallel phasic and tonic systems, then, yields three basic features of adaptation. First, the phasic component generates range shifting behavior; hence, the degree of range shifting varies directly with the contribution of the phasic component. Second, an adapted, purely phasic system is unidirectional (if we exclude the possibility of negative responses) because it gives a measurable response only above the adapting level. Third, since the phasic component adapts to zero, the presence of a nonzero response to the adapting stimulus intensity (i.e., Stevens's terminal adaptation locus; see below) reflects the contribution of the tonic system. Hence, the tonic response component makes the responses of the adapted system bidirectional by permitting reductions below the level produced by the tonic input (Figure 3).

4. In linear coordinates, the range shifts produced by hybrid phasic-tonic systems are parallel at a slope determined by the gain of the phasic system. If output is transformed logarithmically, though, the curved trajectories of Stevens's adaptation curves emerge.

\section{Implications for Sensory Processes}

The underlying thesis of this article is that a simple model based on summation of phasic and tonic systems is sufficient to predict many properties of sensory psychophysical phenomena. Although it would be naive to assume that such a simple model can completely characterize any sensory system, this mechanistic model provides a simple heuristic for explaining both static and dynamic behavior of adaptation, as well as other sensory processes usually viewed as independent phenomena.

\section{Impulse Response}

The response to an instantaneous stimulus is not instantaneous, but is spread over time. The length of time over which the system responds to an impulse (a very intense, instantaneous stimulus), including a positive phase followed by a negative phase, is the memory time of the system. Stimuli that occur at intervals less than the memory time will interact with one another. They may summate or fuse (e.g., critical flicker frequency). Adaptation, then, implies a lack of temporal discrimination. The system will respond to trains at longer intervals without adapting.

\section{Range Shifting}

The stimuli to which many sensory systems respond vary over an extremely wide range of intensities. Envi- 


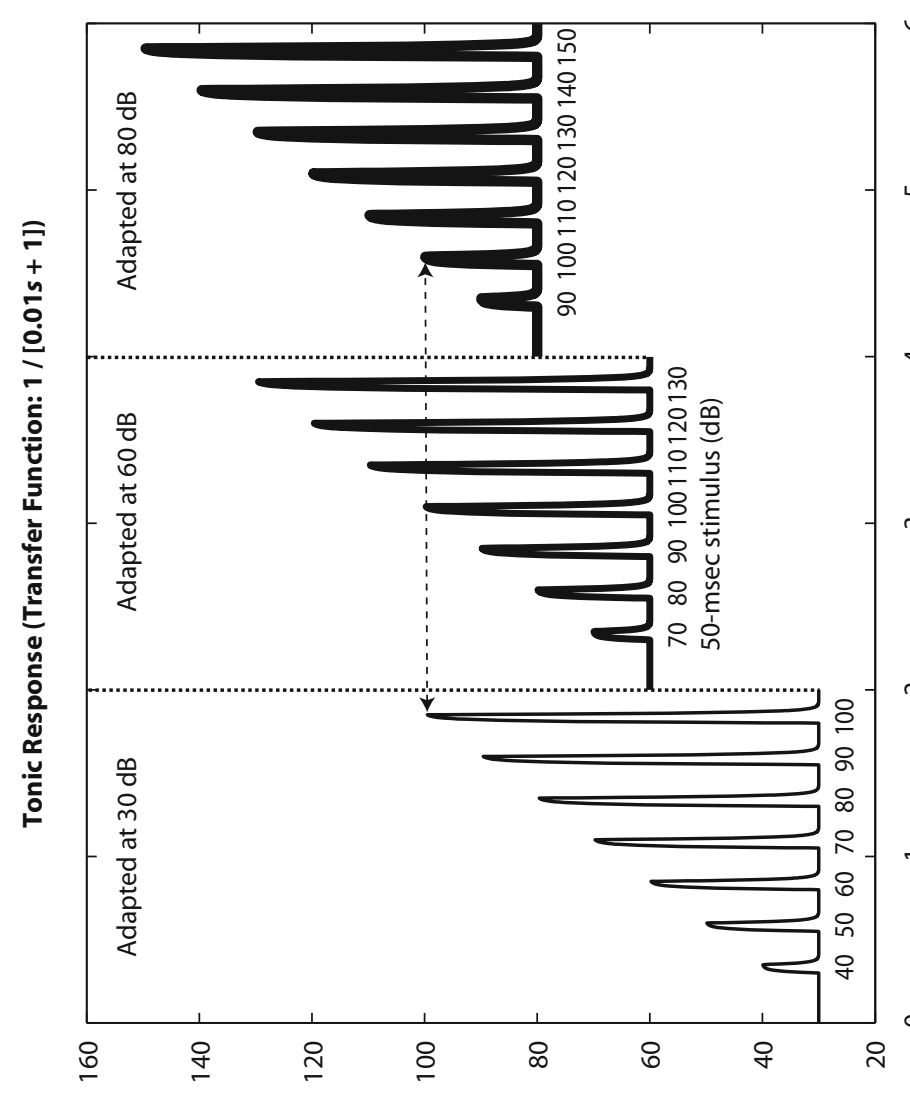

$\infty$

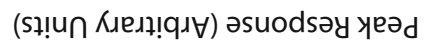

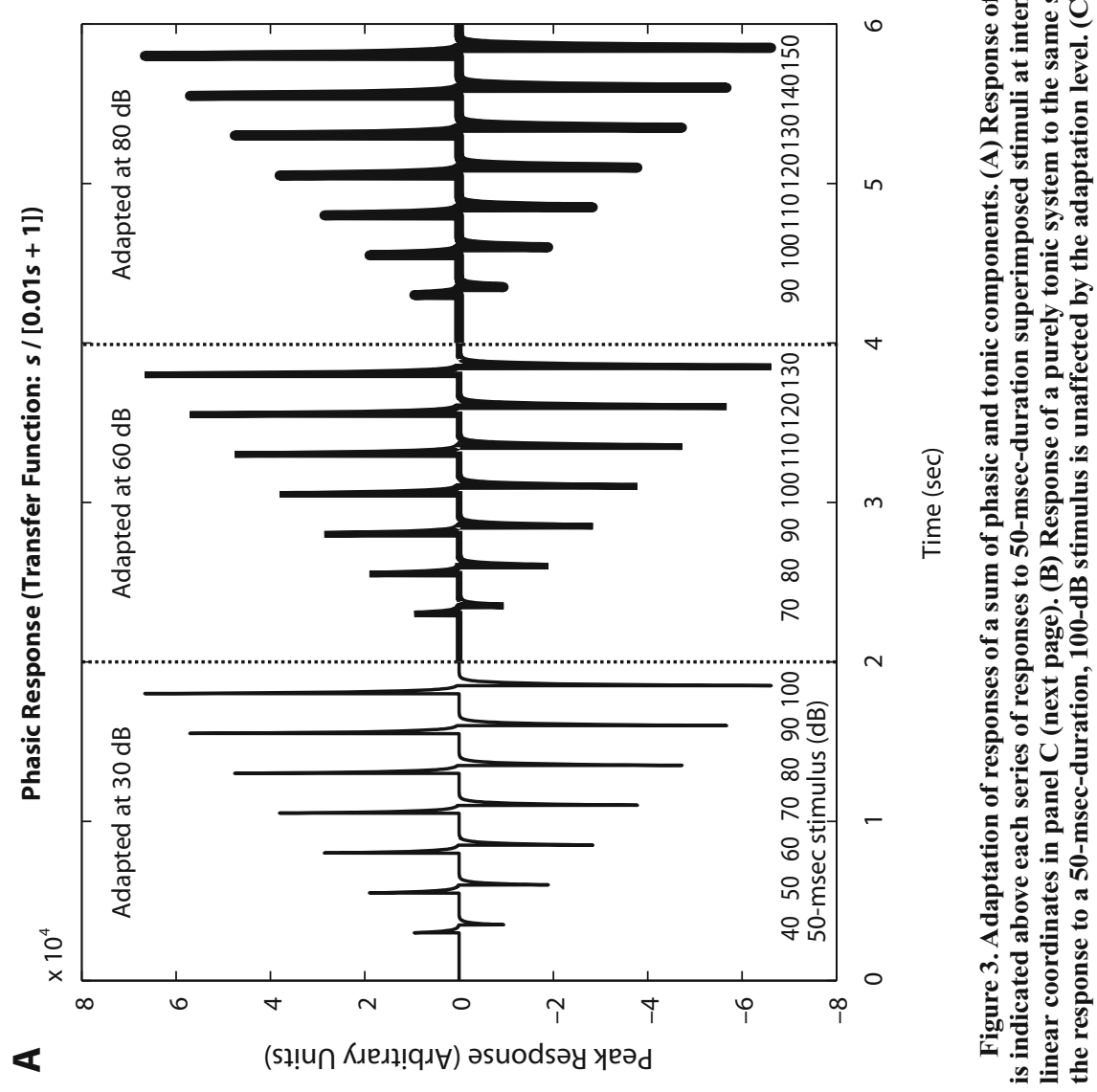



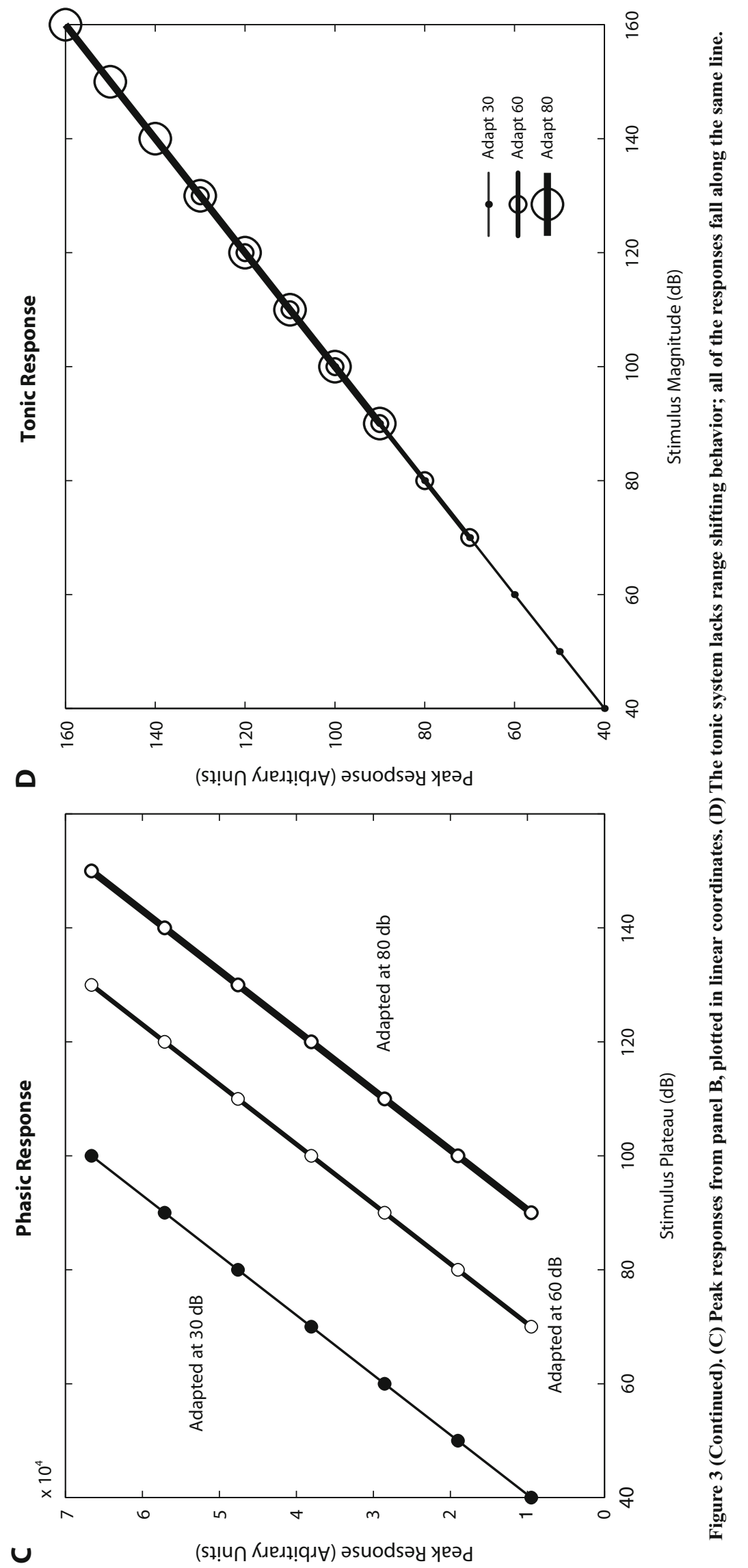
ronmental light intensity, for example, varies by more than 10 orders of magnitude. In order to function effectively, therefore, the eye must have a very wide dynamic range. If the system were required to respond to all 10 orders of magnitude at the same time, and if response were a logarithmic function of intensity (Fechner's law), then a $1 \log$-unit (10:1) increase in the stimulus would increase the response by $10 \%$ of its maximum. If, however, the response were a power function of intensity (Stevens's law), there would be no single corresponding number that applied over the entire range of intensities. But for vision, a 1 log-step increase in intensity in the middle of the range of intensities (measured in $\log$ units) would increase the response, according to Stevens, by about $2.5 \%$ of its maximum; in other words, the sensory system has the problem of spreading its limited sensitivity over a very wide range of intensities.

By range shifting, however, about $50 \%$ of the response range is located within 1 log step of intensity (Shapley \& Enroth-Cugell, 1984). A system that demonstrates range shifting can adjust its behavior to allocate the largest part of its response range to intensities currently being experienced, resulting in an enormous enhancement in performance. The threshold of a completely phasic system, which shows complete adaptation, will lie above the adapting intensity. This is true for taste, which is predominantly phasic (see below), and for temperature, within limits. For systems with substantial tonic components, such as vision and audition - which, therefore, do not show complete adaptation - the threshold is below the level of the adapting stimulus.

\section{Sensitivity Shifting}

Range shifting causes the slope of the response in a particular region of the dynamic range to vary with the intensity of the adapting stimulus. (This is true when the response is plotted against the logarithm of the stimulus, as is general practice.) If one makes the reasonable assumption that the slope of the response as a function of stimulus intensity is related to the ability of the system to discriminate changes in intensity, it follows that the difference threshold would also covary with adaptation, and be smallest near the intensity that serves as the standard intensity.

Two equations commonly used to display sensory intensity functions are the Naka-Rushton equation $\left[R / R_{\max }=\right.$ $I / I+a$, where $a$ is a parameter related to adaptation] and Stevens's power function $\left[R=k\left(I-I_{\mathrm{o}}\right)^{n}\right.$, where $I_{\mathrm{o}}$ is a parameter related to adaptation]. The slopes of both of these equations are influenced by adaptation, being steeper the higher the adapting level. Furthermore, the slope is steepest just above the minimum response. Once again, this is the case when the response is plotted against the logarithm of the stimulus, as is common practice.

The increase in ability to discriminate changes in a given stimulus intensity (that is, a decrease in the difference threshold) when the receptor has been adapted to that stimulus has been observed in a number of senses: auditory (Bekesy, 1929/1960); gustatory (McBurney, Kasschau, \& Bogart, 1967); vibrotactile (Goble \& Hol- lins, 1993); and visual (Greenlee \& Heitger, 1988; Keidel et al., 1961; Ranke, 1952, as cited by Keidel et al., 1961). The widespread occurrence of this phenomenon has been taken to indicate a general principle of sensory processes (Goble \& Hollins, 1993; McBurney \& Collings, 1977).

We should note that Stevens rejects any psychological usefulness of difference thresholds, and would, therefore, reject any attempt to relate the difference threshold to the steepness of slope of magnitude function. We believe that he is too doctrinaire on this point, however. For a system with a phasic component and a logarithmically scaled stimulus, the steepness of the magnitude function decreases with increasing stimulus intensity, relative to the adaptation level. This implies that the difference threshold for a given standard stimulus intensity will be inversely related to its distance from the adapting level. This relationship between difference thresholds and the steepness of the magnitude function has been noted empirically by others (e.g., Krueger, 1989).

\section{Adaptation As a Mechanism for Detecting Changes}

It follows from the phenomena of range shifting and sensitivity shifting that adaptation can be seen as a mechanism for detection of changes in intensity. This may be seen clearly in the case of chemotaxis in bacteria, as described by Berg (1988). E. coli is $10^{-4} \mathrm{~cm}$ in diameter, or $1 / 200,000$ the size of a human. Owing to its small size, this animal cannot use spatial gradients as the basis for orientation toward a food source. It swims in a somewhat curved path for a variable period of time, followed by a tumble, after which it continues swimming in a more or less random direction. In the absence of a gradient of concentration of an attractant (or repellent) its runs average about $1 \mathrm{sec}$. Runs are longer when the organism happens to be swimming up a gradient of attractant. Those that do not result in swimming up a gradient are shorter. This behavioral strategy is known as klinokinesis (Dusenbery, 1989b). Segall, Block, and Berg (1986) modeled klinokinesis by a simple adapting system that adds the stimulus intensity over the last second and subtracts the intensity of the previous $3 \mathrm{sec}$. This system does not respond to steady concentrations, but responds positively to increases and negatively to decreases.

Dusenbery (1989a) developed a model system to test the role of adaptation in klinokinesis. The model tended to move in a straight line as long as the movement resulted in a positive gradient of stimulus intensity. The smaller the gradient, the greater was the probability that the model would make a random change in direction. He found that adaptation improved the efficiency of the model in the presence of three types of noise, each of which is a realistic assumption.

\section{Phase Leading}

The ability of a phasic system to lead the magnitude of a regularly varying stimulus permits the organism to anticipate the maximum stimulus input. This property allows the organism to counteract the lag between receptor and response inevitable in any physiological system, and thereby prevent oscillations in response to disturbances (Pringle 
\& Wilson, 1952). Such a property has been demonstrated for systems as disparate as the phototropic response of the fungus Phycomyces (Foster \& Smyth, 1980) and the muscle spindle of the human hand (Merton, 1951).

Because the vestibular system responds strictly to acceleration and not to velocity, it is a purely phasic system. The vestibular system thus displays phase leading to lowfrequency repeated stimuli, such as sinusoids. This phase leading permits the organism to compensate for response delays between the stimulus and vestibular reflexes (Wilson \& Melvill Jones, 1979).

\section{Fit of the Model to Existing Psychophysical Data}

This section illustrates the ability of the model approach to predict empirical data. We have applied this approach to responses to irritants because their time scale (development over minutes rather than milliseconds) gives the greatest opportunity to characterize time-varying features of the responses. Hence, the focus will be predominantly on new insights into pain psychophysics from this approach. We will then discuss features of existing gustatory, visual auditory, and cutaneous mechanoreceptive system psychophysical data from the perspective of the insights gained from studies of pain.

Pain sensation appears to have tonic, phasic, and integrator (cumulative exposure) components that vary in relative dominance across individuals. Taste appears to be a primarily phasic system, audition appears to be primarily tonic, and vision appears to be intermediate.

Finally, we review recent insights into the relationship between perceived intoxication and blood alcohol levels, which suggest that the approach generalizes beyond boundaries of traditional senses.

The application of this approach to sensory processing requires determination of both the relative contributions of phasic and tonic components to the final responses and the estimation of the parameters of each component. Since the model reflects the dynamic response properties over time, the initial step is to estimate the gains and time constants of the tonic and phasic components from published data that document the full time course of the response. The parameters from these responses are then used to simulate the responses to stimuli that vary in intensity, such as range shifting and Weber's law.

Pain. Psychophysical responses to capsaicin develop over a considerably longer time course than do responses to gustatory, auditory, mechanoreceptive, and visual stimuli. (Because we have done a number of studies on pain with the guidance of the model, we adopt a specific organization for this section.)

Basic properties of the model components. McBurney et al. (1997) asked participants to judge the pain of a fixed concentration of capsaicin applied to the tongue for $34 \mathrm{~min}$. They found that subjects showed one of three different patterns of responses over time: (1) a gradual rise to a maximum at about $10 \mathrm{~min}$, followed by a slow decline for the duration of the stimulation; (2) a similar rise for about $20 \mathrm{~min}$ to a steady state; and (3) a gradual rise that began to level off, but then after about $10 \mathrm{~min}$ continued to rise exponentially for the rest of the session.
These patterns were called phasic, tonic, and integrator, respectively. Using the subjects that showed the typical patterns, McBurney et al. (1997) identified three underlying temporal processes, each having a characteristic time constant. These three processes were then used to describe the data of all subjects, regardless of their pattern. It is significant to note that the same time constants were used for all subjects; thus, the individual differences among subjects could be described as differences in only the gains of the three processes.

From a teleological perspective, the tonic, phasic, and integrator processes define three temporal filters for determining significance of a stimulus. The tonic process is a level detector. Its slow rise time reflects a trade-off of response speed for accuracy in assessing magnitude of a sustained stimulus. The phasic process is a change detector. Rather than being an accurate determination of stimulus intensity, it signals a rate of change of the stimulus; hence, it is specialized for rapid detection. The integrator process is, to our knowledge, unique to irritant stimulation. This process functions as a cumulative exposure detector, which may prevent injurious exposure.

We will discuss individual differences and effects of experience in a later section. We note here that the integrator process is seen for the first time in these data.

Stevens's scaling. The relationship between stimulus intensity $(S)$ and human perception $(R)$ is described by Stevens's power function relation, $R=k S^{n}$. In log-log plots, $n$ is the slope of the function, which is known to differ among the senses. Therefore, we determined the slopes of the tonic, phasic, and integrator processes by presenting differing concentrations (McBurney, Balaban, Popp, \& Rosencranz, 2001). Because the gain is one of the parameters in the transfer function for each concentration, it describes the magnitude of the response curve over its time course. This analysis was performed across all subjects. The tonic process had a slope of about 0.3 , consistent with its role as a level detector over a wide range of concentrations. The integrator and phasic processes both had twice the slope of the tonic, but the phasic saturated. The different slopes of the processes are one indication that the various processes serve different functions. The greater slopes of the phasic and integrator functions suggest that these processes signal danger or damage.

The saturation of the phasic process is consistent with change detection. Because the response peaks rapidly, it is primarily useful in detecting a change at lower intensities. The tonic process, which has a lower slope and no saturation, is consistent with discrimination of stimulus level over a wide range. The integrator process has an intermediate slope and does not saturate, supporting its role as a slow cumulative exposure detector over an expanded range. These differences for processes within the same sensory dimension pain are consistent with differences between different senses. Teghtsoonian (1973) showed that there is a reciprocal relationship between the dynamic range of a stimulus dimension and its slope, such that dimensions with a steep slope have a narrow dynamic range. For example, electric shock has a very high slope of 3.5, whereas visual brightness has a slope of 0.3 . 
Plasticity (longer term exposure). Daily exposure to a moderate $(75 \mathrm{ppm})$ capsaicin concentration modified the relative weights of the response components (McBurney et al., 1997). The tonic and integrator gains (especially the latter) declined over sessions, indicating "adaptation." There was, however, no change in the phasic gain. This is another suggestion that the phasic process permits the system to respond to short-term changes.

It is important to note that the change with exposure occurred only in the weights of the various processes. There was no change in time constants. In other words, the dynamic properties of the processes were not affected by the long-term exposure. Only the relative contribution (gain) of each process seems to be altered in this form of longterm "adaptation."

These changes in response with exposure have been termed, variously, adaptation, habituation, and desensitization. Adaptation is the term most commonly used in the senses. Habituation is generally thought to have effects over a longer time; generally, a matter of days. For this reason, habituation is typically considered to be a type of learning. Desensitization (and the related term sensitization) is typically used to describe the short-term interactions between similar stimuli.

We contend that these three terms describe phenomena predicted by the processes in our model. But the various terms are used somewhat interchangeably in the literature, and the same terms are used for phenomena that result from different processes in our model. For example, adaptation is seen in the overall reduction in the response over time within a single session. This is the result of the temporal properties of the phasic process. But the reduction in response across days, also commonly called "adaptation," results from a change in the gains of the tonic and integrator processes. We believe that our model raises the question of the validity of current usage of these terms; specifically, in the present case, whether adaptation and habituation are actually the same process.

Temporal summation explains adaptation, crossadaptation, sensitization, and cross-sensitization. Sensitization and desensitization are terms commonly used in the pain literature (e.g., Cliff \& Green, 1996). Sensitization describes the situation in which a second stimulus of the same type has a greater effect than the first. Desensitization is used to describe the reduction in response to the second presentation of the same stimulus when they follow one another closely. The latter term appears to be similar to the common usage of the term adaptation. We have shown that both phenomena are predictable consequences of the phasic and tonic response mechanisms to repeated stimulation.

Using intermittent stimulation (two cycles, $10 \mathrm{~min}$ capsaicin/10 min off), Balaban, McBurney, and Stoulis (1999) showed that predictions of the model can describe both sensitization and desensitization (Figure 4). Temporal summation of phasic and tonic components' onresponses was sufficient to reproduce the sensitization of the on-response to the first cycle of stimulation. The same temporal summation process also explained the desensitization to the second cycle of capsaicin stimulation.
Specifically, the response to the second of a pair of stimuli reflects the addition of the tonic response to the second stimulus to the continuing tonic response to the first stimulus, minus the off-response to the first stimulus caused by the phasic process. Desensitization will be seen when the negative-going result of the phasic process to the first stimulus outweighs the positive-going effect of the second stimulus on the tonic process. Thus, desensitization is simply the added effect of the tonic and phasic processes. Sensitization is even simpler: It results from the addition of tonic and phasic processes over successive presentations. Whether sensitization or desensitization is seen is simply a matter of the time between stimuli, and hence relative contributions of the positive- and negativegoing phases of the phasic response relative to the (always) positive-going tonic response.

The implication of the results is clear: "Sensitization" and "desensitization" of oral capsaicin responses refer to predictable consequences of temporal summation of phasic and tonic signals.

Interactions between two irritants. Interactions between pairs of vanilloid irritants, such as capsaicin, piperine, and zingerone, are examples of the ubiquitous phenomenon of cross-adaptation (see, e.g., Goldstein, 2007). Previous investigators (Prescott \& Stevenson, 1996a, 1996b) found that different irritants had differing time courses as well as nonreciprocal patterns of crossadaptation. We have used our model to predict which pairs of irritants will show cross-adaptation (Affeltranger, McBurney, \& Balaban, 2007). For successive stimuli, the responses to zingerone after piperine (Figure $5 \mathrm{~A}$ ), piperine after zingerone (Figure 5B), and capsaicin after piperine behave as a sum of responses to independent presentation of single irritants (Figure 5C).

However, the responses to capsaicin after zingerone were reduced considerably, compared with the predicted additive response of individual exposures to each irritant (Figure 5D). As in the case of response "desensitization" to sequential capsaicin presentations (Balaban et al., 1999), these responses could be explained by an additive model that permits inhibitory actions of the phasic process only for interactions between capsaicin and zingerone.

Individual differences. Pain responses are notorious for their high variability, both between and within subjects. The modeling has been particularly helpful in the investigation of individual differences in dynamics of pain sensation as response phenotypes. The model approach to the question of variability was motivated by the observation that individual differences in normalized free magnitude estimation responses to oral capsaicin could be attributed, in part, to the existence of three different dynamic response patterns. As in our previous work, we found two types of subjects: "level detectors," who showed a tonic type of response, and "change detectors," who showed primarily a phasic response. An unexpected third group, termed "cumulative exposure detectors," reported a burning sensation that rose sharply over time. The rising component of this response behaved as a double integration of the exposure concentration, 
and appeared to add a level detection response component. The shape of the response in level detectors and change detectors was unaltered by a daily exposure to
75 ppm of capsaicin in candy. However, the rising component was abolished by experience, leaving only a level detection response.

A

Model Fit to Median of Data

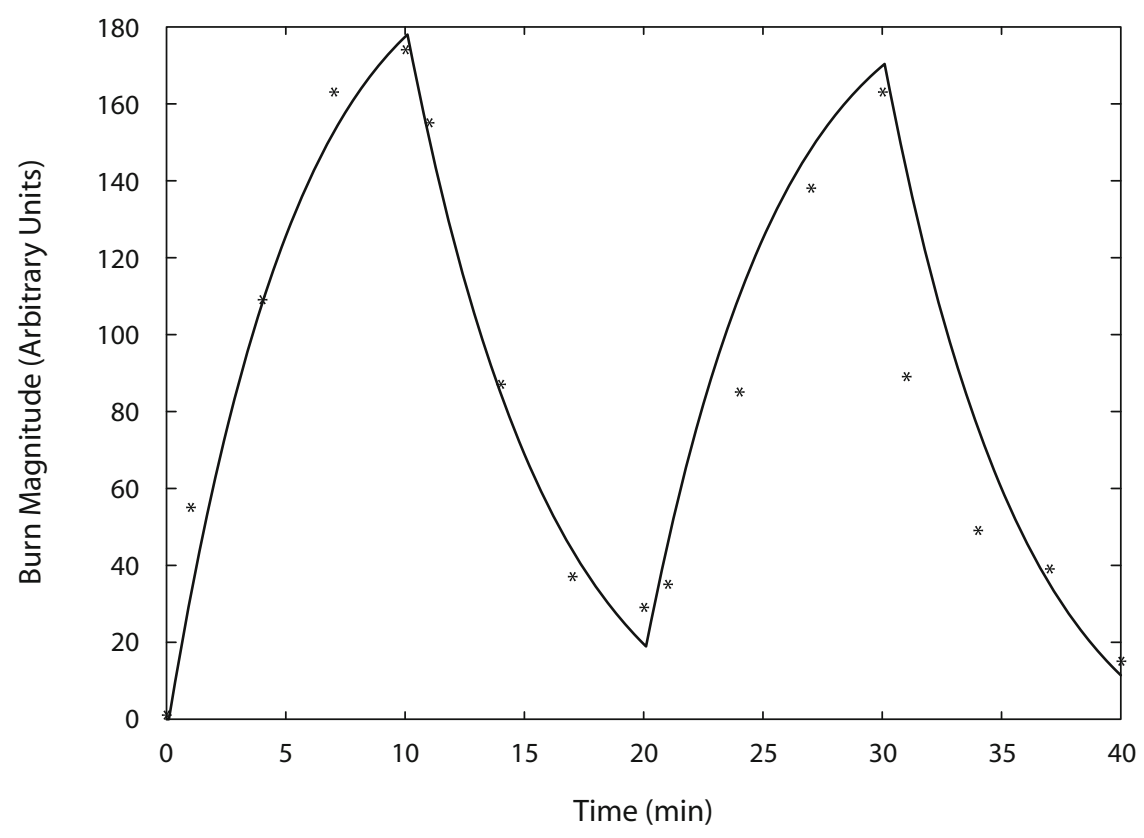

B

Model Components

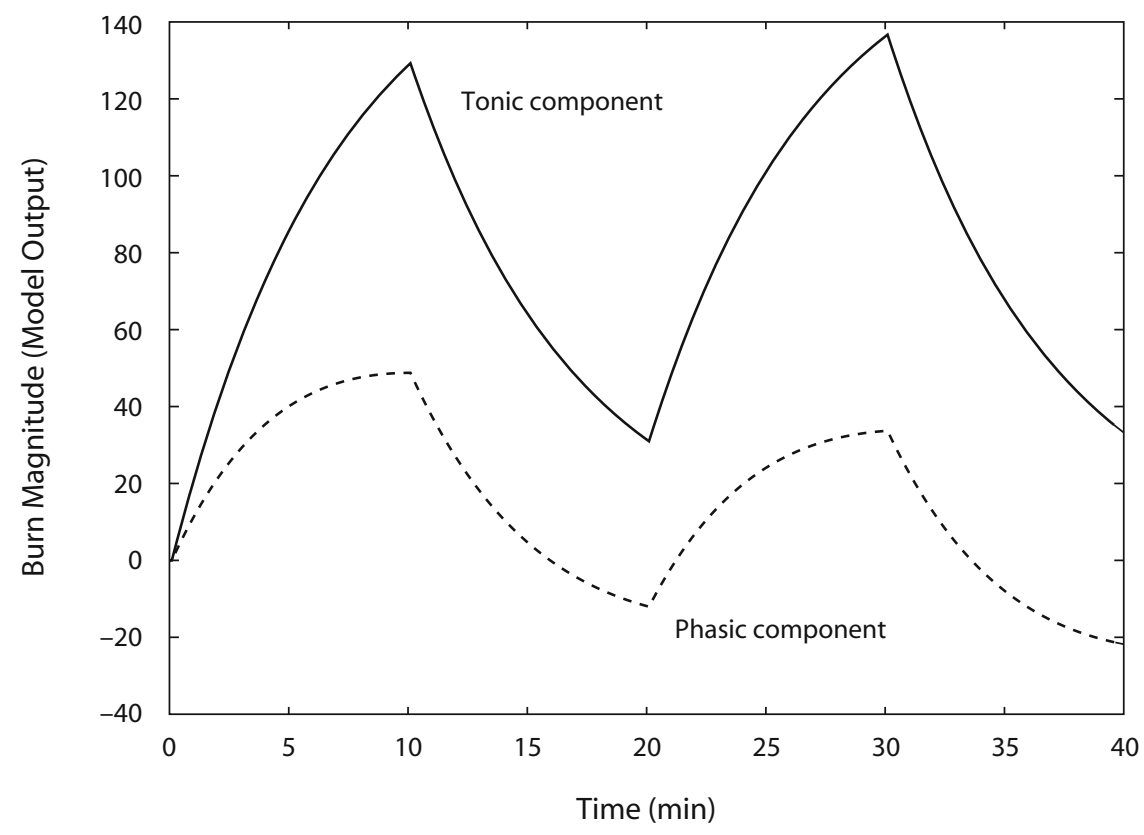

Figure 4. (A) Oral capsaicin was presented for two cycles of $10 \mathrm{~min}$ on and $10 \mathrm{~min}$ off. The line represents the fit of a model with phasic and tonic components to the median of the group data (asterisks). (B) The behavior of the tonic (level detector) and the phasic (change detector) in the model fit from panel $A$ are shown separately. Note that the tonic response to the second cycle is greater than the first, whereas the phasic response is less. The sum of these two components yields the model fit shown in panel A. From "Time Course of Burn to Repeated Applications of Capsaicin," by C. D. Balaban, D. H. McBurney, and M. Stoulis, 1999, Physiology and Behavior, 66, p. 110. Copyright 1999 by Elsevier, Inc. Adapted with permission. 
A

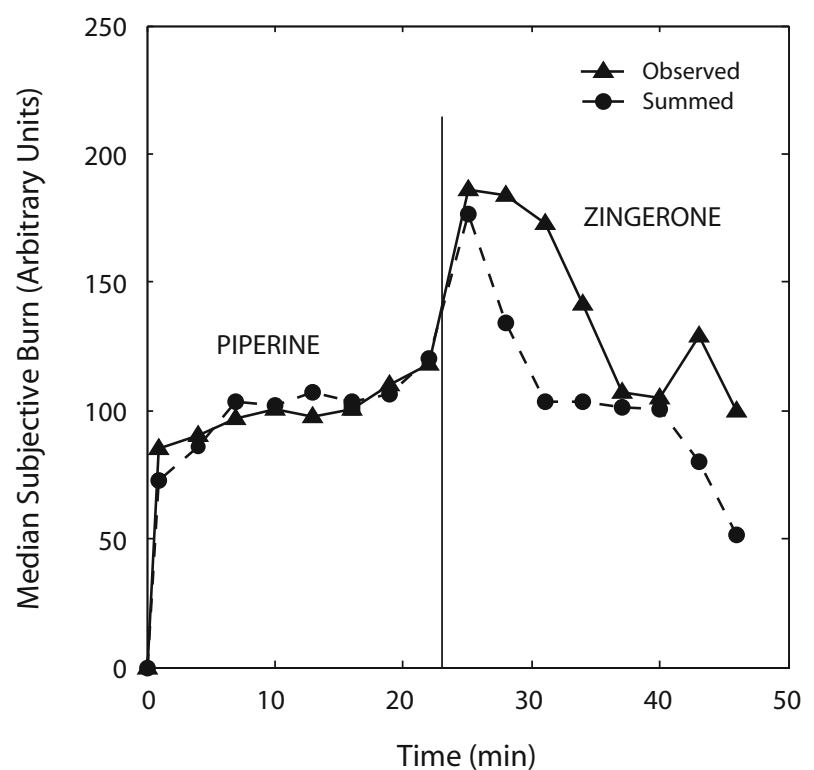

C Piperine Then Capsaicin

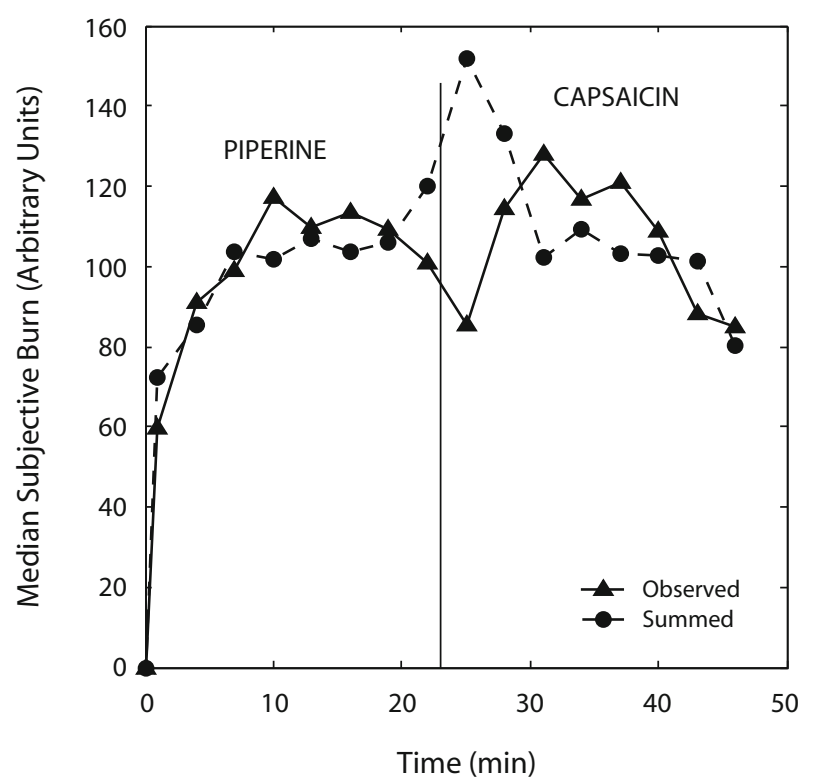

B Zingerone Then Piperine

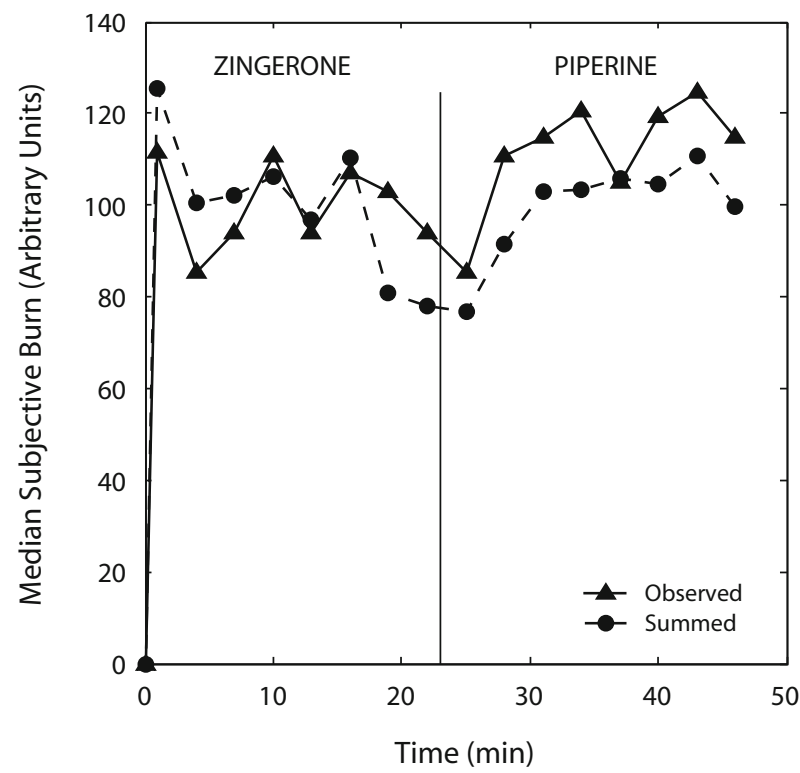

D Zingerone Then Capsaicin

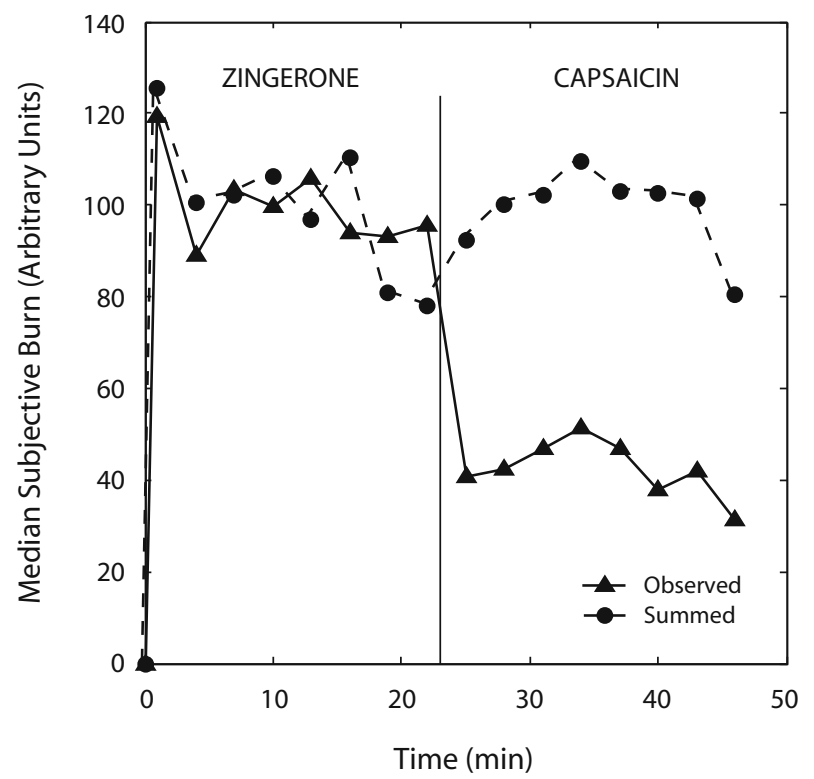

Figure 5. Subjective burn to successive pairs of irritants. The triangles show the data obtained by presenting one irritant immediately after the other. The circles show the results of adding the independent responses to the second irritant to those of the first. The responses for piperine $\rightarrow$ zingerone (A), zingerone $\rightarrow$ piperine (B), and piperine $\rightarrow$ capsaicin (C) show a cross-sensitization that behaves as a temporal summation of the independent responses to presentation of each irritant alone. The response to zingerone $\rightarrow$ capsaicin (D), though, shows a cross-adaptation. From "Temporal Interactions Between Oral Irritants: Application of a Dynamic Model," by M.A. Affeltranger, D. H. McBurney, and C. D. Balaban, 2007, Chemical Senses, 32, p. 460. Copyright 2007 by Oxford University Press. Adapted with permission.

An individual's response pattern (level detection, change detection, or cumulative exposure detection) was remarkably stable in the earlier studies across different stimulus intensities, and for cross-interactions between irritants. Balaban, McBurney, and Affeltranger (2005) tested the hypothesis that the three temporal response phenotypes could be one factor producing the notorious intersubject variability in pain sensation responses. Our mathematical model predicted that two different "two-step" stimulus profiles (ascending step, 30 ppm-300 ppm; descending step, $300 \mathrm{ppm}-10 \mathrm{ppm}$ ) could discriminate the level detection, change detection, and cumulative exposure detection 
A

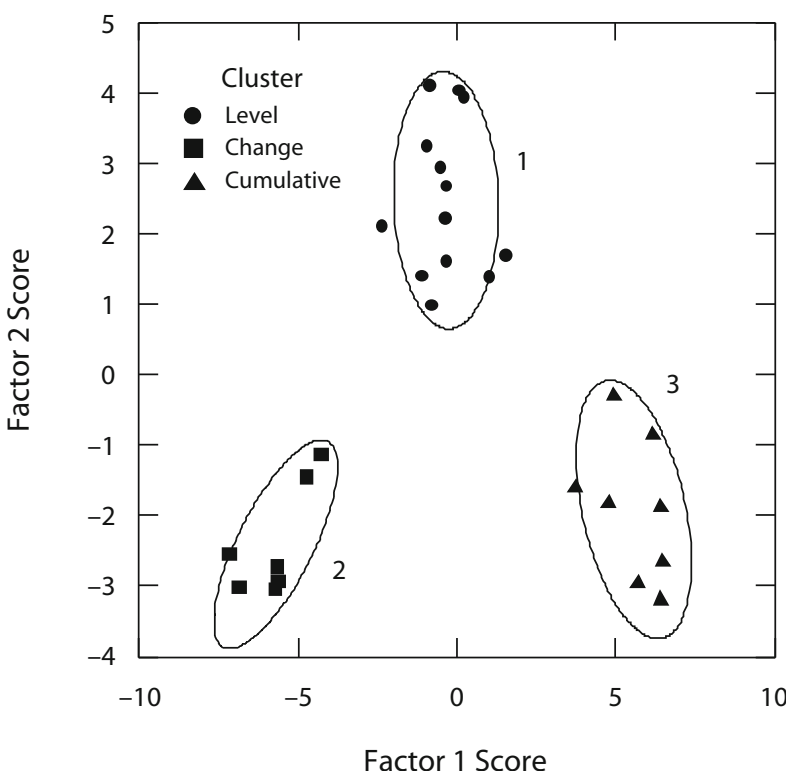

B Ascending Steps

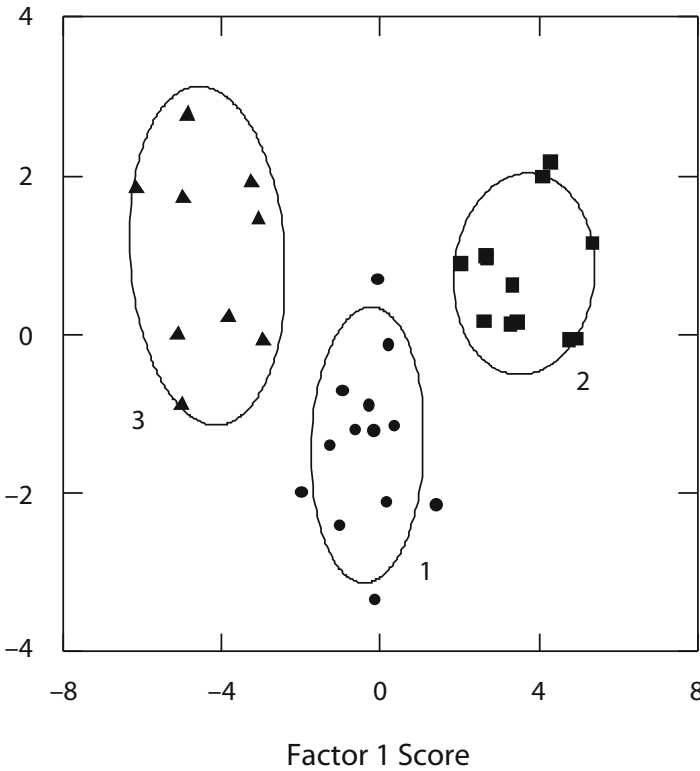

Figure 6. Fuzzy c-means cluster analysis yielded factor scores that classified subjects into three distinct groups, on the basis of their response to a two-step decrease (panel A; 24 min at 300 ppm, followed by 22 min at 10 ppm) or increase (panel B; 15 min at $30 \mathrm{ppm}$, followed by $25 \mathrm{~min}$ at $300 \mathrm{ppm}$ ) in capsaicin concentration on the tongue. The level detectors (Cluster 1), change detectors (Cluster 2), and cumulative irritation detectors (Cluster 3) are indicated by different symbols in each plot. From "Three Distinct Categories of Time Course of Pain Produced by Oral Capsaicin," by C. D. Balaban, D. H. McBurney, and M. A. Affeltranger, 2005, Journal of Pain, 6, p. 321. Copyright 2005 by Elsevier, Inc. Adapted with permission.

responses better than the single step or repeated shorter exposures could. As predicted, fuzzy c-means cluster analysis readily identified three groups of subject responses to both stimulus profiles, corresponding to level detectors, change detectors, and cumulative exposure detectors (Figure 6). This phenotypic classification system confirmed our intuitive classification scheme from the earlier studies.

It is noteworthy that the subject response categories are based entirely on temporal patterns of response, ignoring overall intensity. Sampling subject responses over time is essential for discriminating these groups. For example, an early sampling time will be weighted primarily by the rapid rise of the change detectors, intermediate times will reflect the plateau response of the level detectors, and later responses will reflect the rise in the cumulative response detectors. Hence, any single measurement time introduces variability by mixing inherently heterogeneous response phenotypes.

Sex differences. Male-female differences are another source of systematic variation in perceived pain intensity. For example, Frot, Feine, and Bushnell (2004) demonstrated that the same topical capsaicin stimulus to the cheek or ankle elicits a greater response over time in females than in males. In a subsequent publication (Lariviere, McBurney, Frot, \& Balaban, 2005), we applied our modeling approach to these data.

The shape of the cheek responses conformed to the same model from our oral capsaicin exposure studies; the response profiles included level, change, and cumulative response patterns. The model indicated that the increased female response to cheek stimulation could be explained purely by increased tonic sensitivity. The individual ankle responses were highly variable and delayed, which necessitated introduction of an initial delay operator into the model. In contrast to the cheek data, individuals showed either a cumulative response, or a negligible total response. Despite the superficial differences from the cheek data, the ankle data could also be explained simply as increased tonic sensitivity in the female subjects.

Vision. Kelly (1961a, 1961b, 1962) used critical flicker fusion paradigms to identify the temporal modulation transfer function for visual stimuli in the photopic range. Figure 7A shows the fit provided by a sum of tonic and phasic components to temporal modulation transfer function, in the same configuration as the model for gustation and capsaicin. The tonic component is a first-order lowpass filter; a second-order high-pass filter provided the best fit to this transfer function. The response to a step increase in light intensity from 100 to 1,000 mLambert, or 90 to $100 \mathrm{~dB}$, in units used by Stevens and Stevens (1963) is shown in the upper response of the center panel. After low-pass filtering (Figure 7B, lower curve), it bears a striking resemblance to the response envisioned by Stevens and Stevens (redrawn in Figure 7C).

Stevens and Stevens (1963) measured the brightness of light after adaptation to various intensities. Adaptation in vision affects both the threshold and the growth of brightness above threshold. In response to $10-\mathrm{dB}$ step increases in stimulus luminance, they reported that the apparent brightness "jumps and then settles back to the 

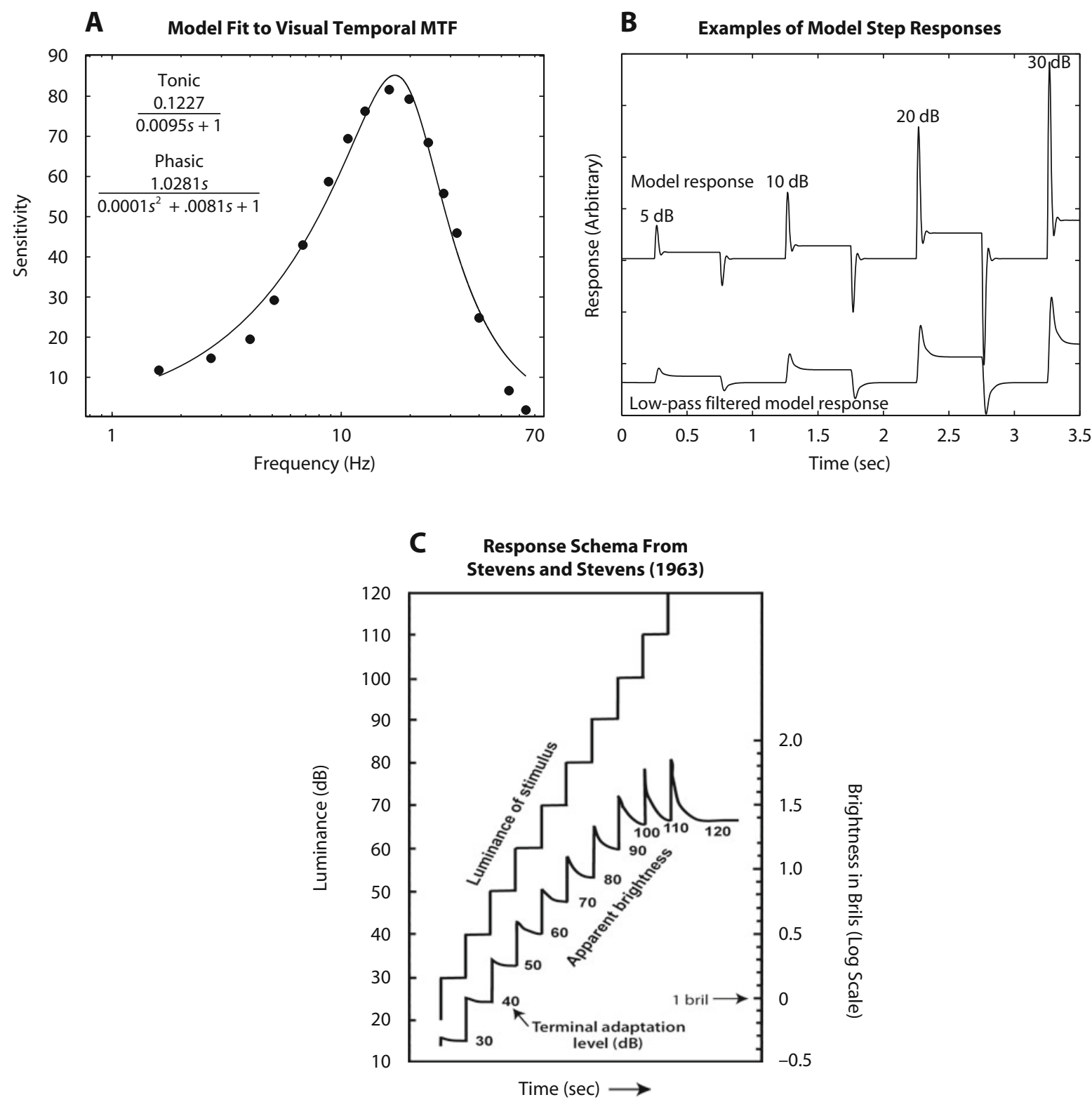

Figure 7. Brightness of light after adaptation to various intensities. (A) Kelly's (1962) visual temporal modulation transfer function (MTF) data from critical flicker fusion frequency studies are plotted, and the data are fitted as a sum of tonic and phasic processes. (B) The step response of the tonic-phasic model, from panel A, is shown above a low-pass filtered representation of the same simulations. Note that the latter simulation result resembles the graph redrawn from Stevens and Stevens (1963) in panel C. See the text for details. From "Brightness Function: Effects of Adaptation," by J. C. Stevens and S. S. Stevens, 1963, Journal of the Optical Society of America, 53, p. 383. Copyright 1963 by the Optical Society of America, Inc. Adapted with permission.

equilibrium level that results when the eye is fully adapted to the luminance of the target" (p. 383). Figure 10 in their article (reproduced here as Figure 7C) is their schematic representation of the "sawtooth function" for apparent brightness during the staircase luminance changes. The final "equilibrium level" for each luminance, across all stimulus presentations, was termed the "terminal brightness function." In our terminology, the terminal brightness function represents the plateau of a tonic (or level detection) process. The rapidly adapting response component represents our phasic (or change detection) process. Thus, the classic Stevens and Stevens data clearly demonstrate that visual brightness adaptation shows considerable contribution of both tonic and phasic components.

Gustation. The temporal modulation transfer functions (McBurney, 1976) and step responses (Gent, 1979) to gustatory stimuli are dominated by phasic-like behavior (Figure 8). The temporal modulation transfer function shows peak sensitivity at approximately $0.1 \mathrm{~Hz}$ for sucrose, $\mathrm{NaCl}$, and citric acid. The step responses show exponential decay (adaptation), with time constants ranging from about 20 to $70 \mathrm{sec}$ (depending on stimulus). Figure 8 shows the results 


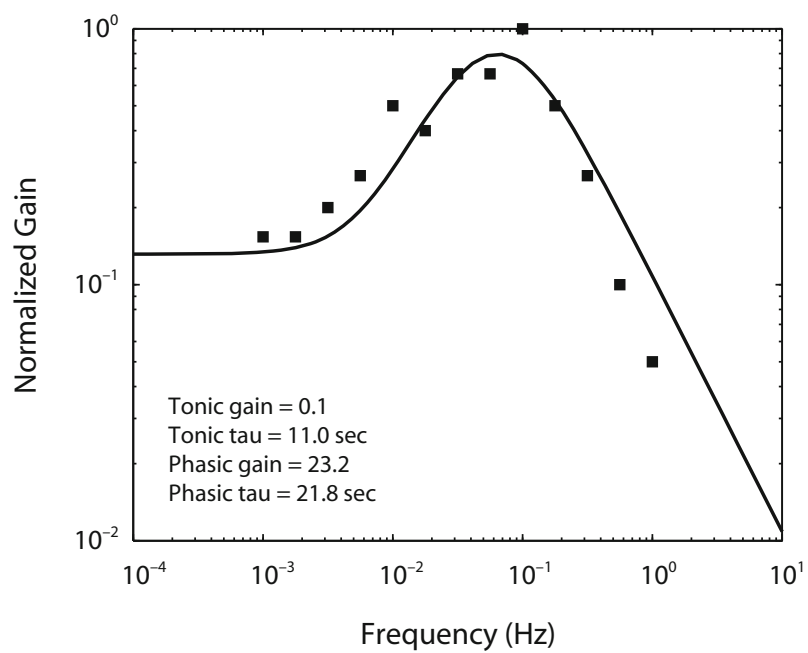

C

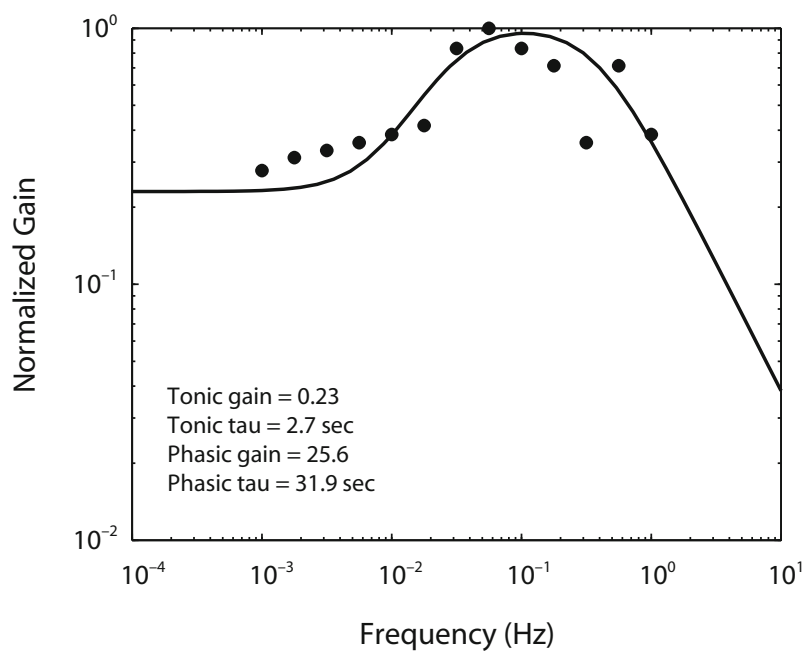

B

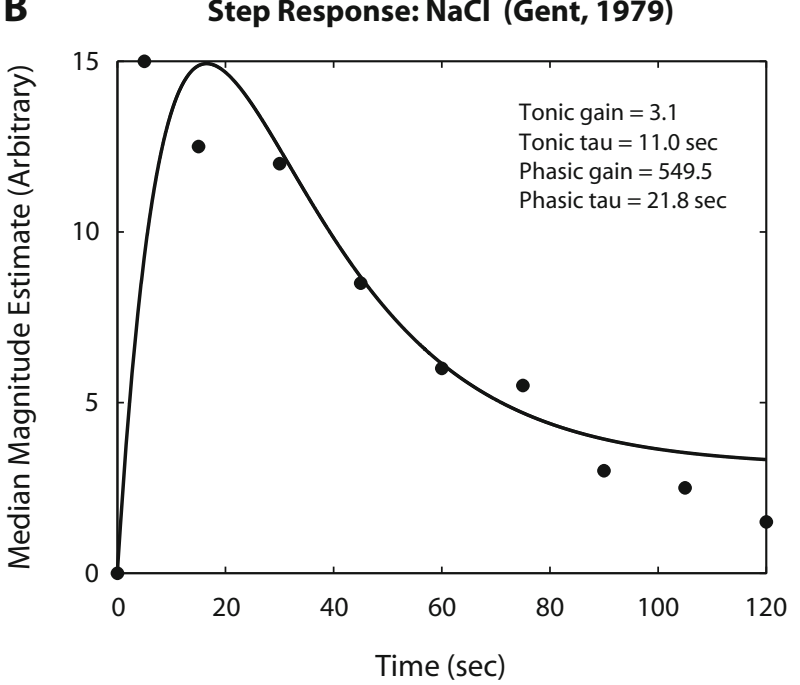

D Step Response: Citric Acid (Gent, 1979)

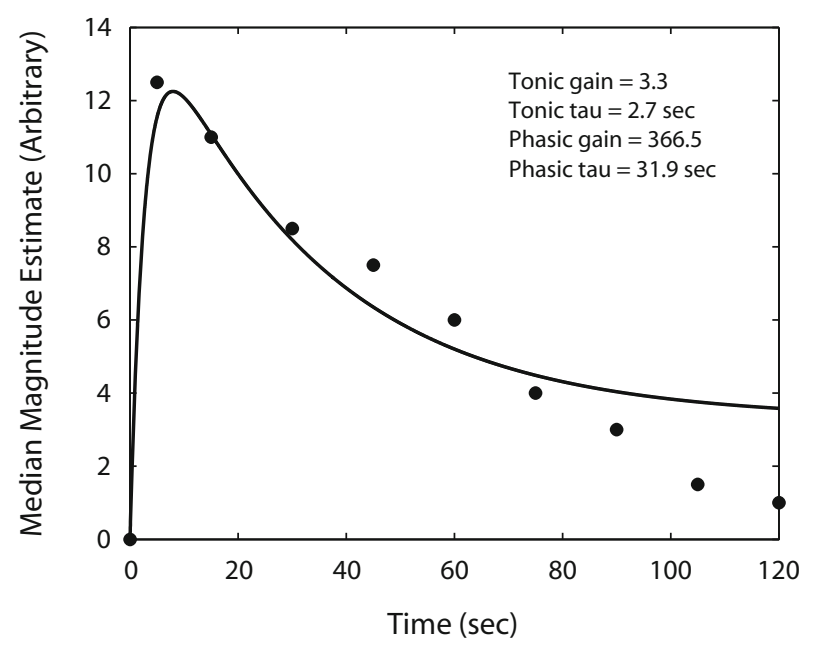

Figure 8. The temporal modulation transfer function (MTF) data points for threshold detection of a sine wave application of $\mathrm{NaCl}$ (A) or citric acid (B) solution on the tongue are replotted from McBurney (1976), paired with the results of Gent's (1979) magnitude estimation data $(C$ and $D$, respectively) in response to a constant concentration (step response, strong stimulus-large area condition). The solid lines represent the fit of the data to the sum of tonic and phasic components. Note that the set of time constants (and ratio of gains) was used for fitting the MTF and step response to $\mathrm{NaCl}(\mathrm{A}$ and $\mathrm{B}$ ) and to citric acid (C and D). See the text for details. From "An Exponential Model for Adaptation in Taste," by J. F. Gent, 1979, Sensory Processes, 3, p. 307. Copyright 1979 by Academic Press. Adapted with permission. From “Temporal Properties of the Human Taste System," by D. H. McBurney, 1976, Sensory Processes, 1 , p. 155. Copyright 1976 by Academic Press. Adapted with permission.

of simultaneously fitting the step responses and transfer functions for either $\mathrm{NaCl}$ (Figures $8 \mathrm{~A}$ and $8 \mathrm{~B}$ ) or citric acid (Figures $8 \mathrm{C}$ and $8 \mathrm{D}$ ) stimuli as the sum of a tonic process with a short time constant $(11.0 \mathrm{sec}$ for $\mathrm{NaCl}$ or $2.7 \mathrm{sec}$ for citric acid) and a longer time constant for the phasic process (21.8 sec for $\mathrm{NaCl}$ and $31.9 \mathrm{sec}$ for citric acid). The transfer function was obtained with a threshold method, and the step response was obtained with magnitude estimation. Nevertheless, each pair of responses to a stimulant can be explained by a single tonic-phasic model with the same time constants. Since the concentration of the stimulus in filter paper in the Gent experiment remained constant during the time of presentation, it is clear that the time course of the response reflects predominantly a phasic process. This observation is reflected in the model result that the phasic gain is two orders of magnitude greater than the tonic gain. Hence, the prototype tonic-phasic model (Figure 1) is sufficient to reproduce the basic temporal properties of responses to gustatory stimuli.

Audition. The degree of auditory adaptation depends greatly on the method of stimulation and measurement, owing to the presence of sizable interaural interactions (Miskiewicz, Scharf, Hellman, \& Meiselman, 1993; Scharf, 1991). Nevertheless, it is possible to say that auditory adaptation is considerably less than in most other senses. Scharf (1983) measured the magnitude estimate 
Temporal MTF From Viemeister (1979)

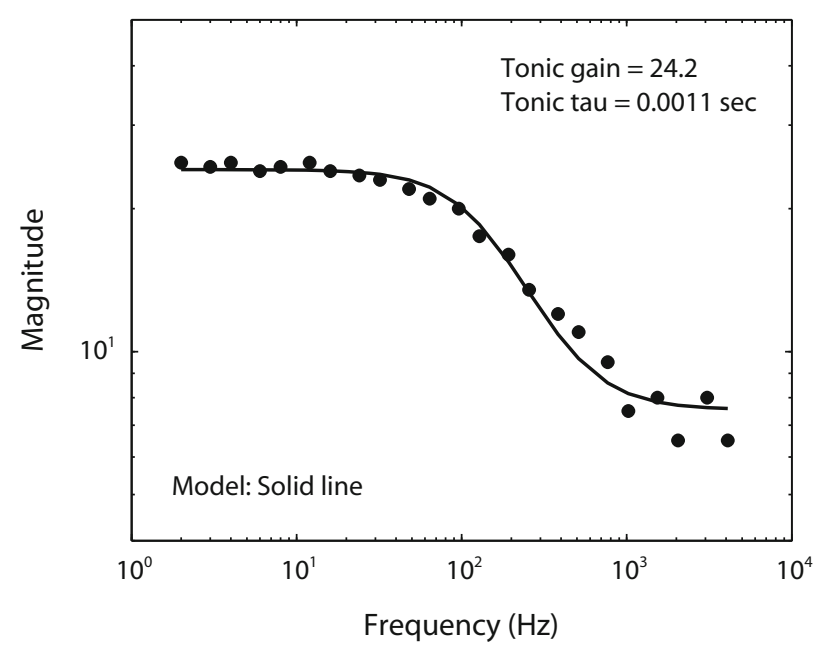

Figure 9. (A) Graph of the fit of a model to the auditory temporal modulation transfer function (MTF) data points published by Viemeister (1979). A tonic component was sufficient to fit the low-pass characteristics of the response. This low-pass transfer function has been described as a sum of a bank of octave-centered, band-pass modulation filters (Dau, Kollmeier, \& Kohlrausch, 1997; Ewert \& Dau, 2000). Examples of these filters are shown in panel B with model fits to each. From "Temporal Modulation Transfer Functions Based Upon Modulation Thresholds," by N. F. Viemeister, 1979, Journal of the Acoustical Society of America, 66, p. 1368. Copyright 1979 by the Acoustical Society of America, Inc. Adapted with permission.

over time of the loudness of tones presented monaurally or binaurally. Monaural presentation resulted in substantial adaptation at $20 \mathrm{~dB}$, less at $40 \mathrm{~dB}$, and none at $70 \mathrm{~dB}$. Binaural stimulation produced less adaptation.

Viemeister (1979) reported that the auditory temporal modulation transfer function to sinusoidal stimuli was dominated by a low-pass filter characteristic. The data (Figure 9A) are fitted well by a first-order tonic process; thus, one could conclude that a tonic process is dominant in audition, with little contribution of a phasic process.

More recent studies (e.g., Dau, Kollmeier, \& Kohlrausch, 1997; Ewert \& Dau, 2000) have used analyses of perception of amplitude modulation to argue that Viemeister's (1979) low-pass characteristic reflects a sum of responses from a bank of modulation filters centered on different octaves. Each of these filters has a band-pass form similar to the visual and gustatory temporal modulation transfer function, which can be described equivalently as a sum of tonic and phasic mechanisms (Figure 9B); hence, a sum of phasic and tonic processes is sufficiently powerful to explain the experimental results and proposed underlying mechanisms. Other adaptive phenomena, such as induced loudness reduction and loudness recalibration, timedependent changes in loudness perception after exposure to a strong tone (Arieh, Kelly, \& Marks, 2005; Arieh \& Marks, 2003), may represent interactions between tonic and phasic response properties of filters representing processing in adjacent octave-centered cochlear regions.

Tactile sensation. Considerable effort has been devoted to elucidating the contributions of cutaneous mechanoreceptors to the sensation of touch (Bolanowski, Gescheider, Verrillo, \& Checkosky, 1988; Johnson, 2001; Leung, Bensmaïa, Hsiao, \& Johnson, 2005; Mountcastle, LaMotte, \& Carli, 1972; Verrillo, 1985). Pacinian corpuscle afferents, rapidly adapting afferents from Meissner's corpuscles, slow adapting afferents (Type 1) from Merkel cells, and slowly adapting afferents (Type 2) from Ruffini endings each respond over distinctive frequency ranges and contribute to dynamic aspects of tactile perception. Differences in their distribution and relative density have been associated with differences in tactile sensitivity of different regions of skin (e.g., lip, cheek, tongue, and fingertip). Recent studies have

\section{Tactile Temporal MTF}

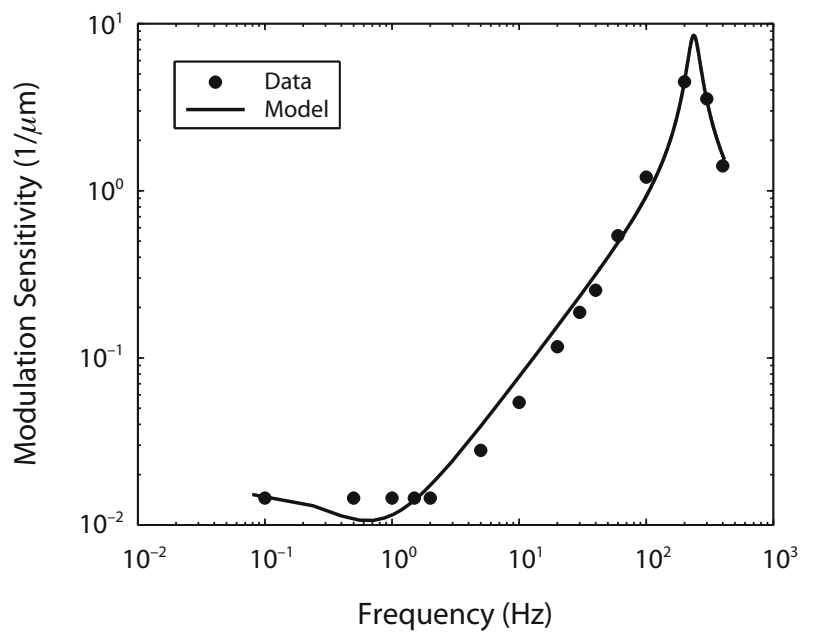

Figure 10. This graph shows the frequency domain behavior of the tactile temporal modulation transfer function data (Data) and the fit provided by a sum of phasic and tonic components (Model). The transfer functions for the components are as follows. Tonic $=0.248 /(s+1.6183)$ and Phasic $=2,705.4 s /$ $\left(s^{2}+317.86 s+2.2199 \times 10^{6}\right)$. 
also suggested that each receptor class has distinct functions in tactile perception of the hand (Johnson, 2001; Johnson, Yoshioka, \& Vega-Bermudez, 2000). We will restrict our discussion to showing that the behavior of the temporal modulation transfer function for tactile stimulation can also be represented heuristically as the sum of a phasic (change detector) and a tonic (level detector) system.

Figure 10 shows the frequency domain behavior of thresholds for human fingertip vibrotactile sensation from above $2 \mathrm{~Hz}$ (Mountcastle et al., 1972), supplemented by the lower frequency response characteristics reported in later studies (Bolanowski et al., 1988). The data are also fitted well as a sum of phasic and tonic processes. It should be noted that the data for the fingertip have a large contribution from Pacinian corpuscle afferents, which are distributed relatively densely in the glabrous skin of the fingers (Johnson, 2001; Mountcastle et al., 1972). Given the elevated density of Pacinian corpuscles in the palm, which have peak sensitivity in the range of a few hundred hertz, one would predict an elevated phasic gain relative to the fingertip. On the basis of the transfer functions of each class of cutaneous afferent (e.g., Bolanowski et al., 1988), it seems reasonably certain that the temporal aspects of tactile sensation can be expressed for any skin region as a sum of tonic and phasic components.

\section{Interoception}

Interoception can be defined restrictively as awareness of visceral sensation or more broadly as effect of visceral sensory activity on molar organismal behavior, even in the absence of awareness (Cameron, 2002). Perceived alcohol intoxication is therefore within the realm of both definitions of interoception; hence, it is significant to note that a sum of tonic and phasic processes is sufficient to explain the large individual variations in the subjective response to alcohol within bouts of drinking and is correlated with self-reported alcohol consumption patterns (Martin, Balaban, \& McBurney, 2006).

The subjective intoxication follows the phasic component of the model (with a decay time constant of $2 \mathrm{~h}$ ) considerably more than does the tonic component, which indicates that it is change in blood alcohol concentration in the individual, not concentration per se, that is a major determinant of subjective intoxication. Furthermore, the relative stability of phasic and tonic gains in an individual implies that variations in the kinetics of alcohol metabolism across drinking bouts are a major determinant of variations in an individual's subjective intoxication. There also were interesting differences between genders, between those with different drinking histories, and between those with and without family histories of alcoholism. Women tended to show higher phasic response and lower tonic response than did men, indicating that women more than men respond to the rising blood alcohol concentration. Furthermore, the phasic gain of males decreased significantly across drinking sessions, but did not change in females. Finally, phasic gains showed significant negative correlations with drinking frequency and drinks per week in those with a negative family history of alcoholism. However, individuals with a positive family history of alcoholism showed no such correlations. These findings suggest that the dynamics of phasic and tonic components of perceived intoxication may have different impacts on alcohol consumption behavior; thus, a sum of phasic and tonic processes has heuristic value for the temporal dynamics of sensations related to exteroceptive and interoceptive sensory modalities.

Recent concepts regarding the neural substrates for interoception, subjective feeling, and emotional awareness suggest that our heuristic parsing of sensations into phasic and tonic components may have applications beyond traditional sensory psychophysics. Craig (2009) has recently reviewed evidence that the insular cortex contains interoceptive representations and is important for placing those representations within motivational, social, and cognitive contexts, and for emotional awareness. Within this framework, it is reasonable to consider the likelihood that some dynamic aspects of motivational and emotional expression may have phasic and tonic components. In fact, similar patterns of dynamic behavior formed the core of the Solomon and Corbit (1974) opponent-process model of motivation. This very influential model of habituation of emotion has also been applied to the process of drug tolerance (e.g., Koob, 2004; Solomon, 1977). The Solomon and Corbit model postulates two processes that differ in both onset time and polarity. Although the mathematical form of the processes is never stated formally, the conceptual figures have the shape of a sum of our phasic and tonic processes. In fact, the classic Solomon and Corbit figures represent the special case of a response of a sum of phasic and tonic components to a square wave (brief constant) stimulus. To our knowledge, this important model has only been used schematically to illustrate properties of data; it has never been implemented explicitly (mathematically) to predict and fit experimental data. Nonetheless, it is important to acknowledge its importance in spurring the field to recognize similarities between the temporal dynamics of interoceptive and exteroceptive sensations, emotional awareness, and motivational awareness.

\section{Sensory Adaptation As a Selective Advantage}

The term adaptation in general refers to a process that adjusts to circumstances in a way that benefits the organism (Tooby \& Cosmides, 1992). Hence, it does not seem to be an accident of language that the word adaptation is used to refer to certain properties of the senses and also to modifications within an evolutionary time scale. The widespread evidence for sensory adaptation in organisms from bacteria to humans further suggests that the integration of phasic and tonic components is a highly conservative, highly efficacious solution to a ubiquitous biological challenge. Adaptation is, therefore, a prominent feature of Mother Nature's small bag of tricks.

\section{AUTHOR NOTE}

Correspondence concerning this article should be addressed to C. D. Balaban, Department of Otolaryngology, University of Pittsburgh, 200 Lothrop Street, Pittsburgh, PA 15213 (e-mail: cbalaban@pitt.edu). 


\section{REFERENCES}

AdRIAN, E. D. (1928). The basis of sensation. New York: W. W. Norton. Affeltranger, M. A., McBurney, D. H., \& Balaban, C. D. (2007). Temporal interactions between oral irritants: Application of a dynamic model. Chemical Senses, 32, 455-462.

Arieh, Y., Kelly, K., \& Marks, L. E. (2005). Tracking time to recovery after induced loudness reduction. Journal of the Acoustical Society of America, 117, 3381-3384.

ArieH, Y., \& MARKs, L. E. (2003). Time course of loudness recalibration: Implications for loudness enhancement. Journal of the Acoustical Society of America, 114, 1550-1556.

Balaban, C. D., McBurney, D. H., \& Affeltranger, M. A. (2005). Three distinct categories of time course of pain produced by oral capsaicin. Journal of Pain, 6, 315-322.

Balaban, C. D., McBurney, D. H., \& Stoulis, M. (1999). Time course of burn to repeated applications of capsaicin. Physiology \& Behavior, 66, 109-112.

BeKESY, G. vON (1960). Experiments in hearing. New York: McGrawHill. (Original work published 1929)

Berg, H. C. (1988). A physicist looks at bacterial chemotaxis. Cold Spring Harbor Symposia on Quantitative Biology, 53, 1-9.

Bolanowski, S. J., JR., Gescheider, G. A., Verrillo, R. T., \& CheCKOSKY, C. M. (1988). Four channels mediate the mechanical sense of touch. Journal of the Acoustical Society of America, 84, 1680-1694.

BrücK, K., Baum, E., \& Schwennicke, H. P. (1976). Cold-adaptive modifications in man induced by repeated short-term cold-exposures and during a 10-day and -night cold-exposure. Pflügers Archives, $\mathbf{3 6 3}$, 125-133.

CAmeron, O. G. (2002). Visceral sensory neuroscience. New York: Oxford University Press.

Cliff, M. A., \& Green, B. G. (1996). Sensitization and desensitization to capsaicin and menthol in the oral cavity: Interactions and individual differences. Physiology \& Behavior, 59, 487-494

Craig, A. D. (2009). How do you feel-now? The anterior insula and human awareness. Nature Reviews Neuroscience, 10, 59-70.

Critchley, M. (1986). The citadel of the senses. New York: Raven Press.

DALtON, P., \& WySOcKI, C. J. (1996). The nature and duration of adaptation following long-term odor exposure. Perception \& Psychophysics, 58, 781-792.

Dau, T., Kollmeier, B., \& Kohlrausch, A. (1997). Modeling auditory processing of amplitude modulation: II. Spectral and temporal integration. Journal of the Acoustical Society of America, 102, 29062919.

DUSENBERY, D. B. (1989a). Efficiency and the role of adaptation in klinokinesis. Journal of Theoretical Biology, 136, 281-293.

DUSENBERY, D. B. (1989b). The value of asymmetric signal processing in klinokinesis. Biological Cybernetics, 61, 401-404.

Dworkin, B. R. (1993). Learning and physiological regulation. Chicago: University of Chicago Press.

EwERT, S. D., \& DAU, T. (2000). Characterizing frequency selectivity for envelope fluctuations. Journal of the Acoustical Society of America, 108, 1181-1196.

Foster, K. W., \& SMYth, R. D. (1980). Light antennas in phototactic algae. Microbiological Reviews, 44, 572-630.

Frot, M., Feine, J. S., \& Bushnell, M. C. (2004). Sex differences in pain perception and anxiety. A psychophysical study with topical capsaicin. Pain, 108, 230-236.

Geldard, F. A. (1968). A note on "adaptation" and allied terms. In D. R. Kenshalo (Ed.), The skin senses (pp. 593-594). Springfield, IL: Charles C. Thomas.

Gent, J. F. (1979). An exponential model for adaptation in taste. Sensory Processes, 3, 303-316.

Goble, A. K., \& Hollins, M. (1993). Vibrotactile adaptation enhances amplitude discrimination. Journal of the Acoustical Society of America, 93, 418-424.

Goldstein, E. B. (2007). Sensation and perception (7th ed.). Pacific Grove, CA: Wadsworth.

GreenleE, M. W., \& Heitger, F. (1988). The functional role of contrast adaptation. Vision Research, 28, 791-797.

Hensel, H. (1968). Discussion. In D. R. Kenshalo (Ed.), The skin senses (p. 328). Springfield, IL: Charles C. Thomas.
JoHnson, K. O. (2001). The roles and functions of cutaneous mechanoreceptors. Current Opinion in Neurobiology, 11, 455-461.

Johnson, K. O., Yoshioka, T., \& Vega-Bermudez, F. (2000). Tactile functions of mechanoreceptive afferents innervating the hand. Journal of Clinical Neurophysiology, 17, 539-558.

Karrer, T., \& BartoshuK, L. (1991). Capsaicin desensitization and recovery on the human tongue. Physiology \& Behavior, 49, 757764.

Keidel, W. D., Keidel, U. O., \& Wigand, M. E. (1961). Adaptation: Loss or gain of sensory information? In W. A. Rosenblith (Ed.), Sensory communication (pp. 319-338). Cambridge, MA: MIT Press.

KeLly, D. H. (1961a). Visual responses to time-dependent stimuli: I. Amplitude sensitivity measurements. Journal of the Optical Society of America, 51, 422-429.

Kelly, D. H. (1961b). Visual responses to time-dependent stimuli: II. Single-channel model of the photopic visual system. Journal of the Optical Society of America, 51, 747-754.

Kelly, D. H. (1962). Visual responses to time-dependent stimuli: III. Individual variations. Journal of the Optical Society of America, $\mathbf{5 2}, 89-95$

KIANG, N. Y.-S. (1966). Discharge patterns of single fibers in the cat's auditory nerve. Cambridge, MA: MIT Press.

Kimble, G. A. (1990). Mother nature's bag of tricks is small. Psychological Science, 1, 36-41.

КоОв, G. F. (2004). Allostatic view of motivation: Implications for psychopathology. In M. A. Bevins \& M. T. Bardo (Eds.), Motivational factors in the etiology of drug abuse. Nebraska symposium on motivation (Vol. 50, pp. 1-18). Lincoln: University of Nebraska Press.

Koshland, D. E., JR. (1980). Bacterial chemotaxis as a model behavioral system. New York: Raven Press.

KRUEger, L. E. (1989). Reconciling Fechner and Stevens: Toward a unified psychophysical law. Behavioral \& Brain Sciences, 12, 251-320.

Lariviere, W. R., McBurney, D. H., Frot, M., \& Balaban, C. D. (2005). Tonic, phasic, and integrator components of psychophysical responses to topical capsaicin account for differences of location and sex. Journal of Pain, 6, 777-781.

Leung, Y. Y., Bensmaïa, S. J., Hsiao, S. S., \& Johnson, K. O. (2005). Time-course of vibratory adaptation and recovery in cutaneous mechanoreceptive afferents. Journal of Neurophysiology, 94, 3037-3045.

Loewenstein, W. R. (1971). Mechano-electric transduction in the Pacinian corpuscle. Initiation of sensory impulses in mechanoreceptors. In W. R. Loewenstein (Ed.), Handbook of sensory physiology (Vol. 1, pp. 269-290). Berlin: Springer.

Martin, C. S., Balaban, C. D., \& McBurney, D. H. (2006). Tonic and phasic processes in the acute effects of alcohol. Experimental \& Clinical Psychopharmacology, 14, 209-218

McBurney, D. H. (1976). Temporal properties of the human taste system. Sensory Processes, 1, 150-162.

McBurney, D. H., Balaban, C. D., Christopher, D. E., \& Harvey, C. (1997). Adaptation to capsaicin within and across days. Physiology \& Behavior, 61, 181-190.

McBurney, D. H., Balaban, C. D., Popp, J., \& Rosencranz, J. (2001) Adaptation to capsaicin burn: Effects of concentration and individual differences. Physiology \& Behavior, 72, 205-216.

McBurney, D. H., \& Collings, V. B. (1977). Introduction to sensation/ perception. Englewood Cliffs, NJ: Prentice Hall.

McBurney, D. H., Kasschau, R. A., \& Bogart, L. M. (1967). The effect of adaptation on taste jnds. Perception \& Psychophysics, 2, 175 178

Merton, P. A. (1951). The silent period in a muscle of the human hand Journal of Physiology, 114, 183-198.

Miskiewicz, A., Scharf, B., Hellman, R., \& Meiselman, C. (1993). Loudness adaptation at high frequencies. Journal of the Acoustical Society of America, 94, 1281-1286.

Mountcastle, V. B., LaMotte, R. H., \& Carli, G. (1972). Detection thresholds for stimuli in humans and monkeys: Comparison with threshold events in mechanoreceptive afferent nerve fibers innervating the monkey hand. Journal of Neurophysiology, 35, 122-136.

Partridge, L. D., \& Glaser, G. H. (1960). Adaptation in regulation of movement and posture. Journal of Neurophysiology, 23, 257-268.

Partridge, L. D., \& Partridge, L. D. (1993). The nervous system: Its function and its interaction with the world. Cambridge, MA: MIT Press. 
Pashler, H. (ED. IN CHIEF) (2002). Stevens' Handbook of experimental psychology (3rd ed., 4 vols.). New York: Wiley.

PenN, J. S., \& Williams, T. P. (1986). Photostasis: Regulation of daily photon-catch by rat retinas in response to various cyclic illuminances. Experimental Eye Research, 43, 915-928.

Prescott, J., \& Stevenson, R. J. (1996a). Desensitization to oral zingerone irritation: Effects of stimulus parameters. Physiology \& Behavior, 60, 1473-1480.

Prescott, J., \& Stevenson, R. J. (1996b). Psychophysical responses to single and multiple presentations of the oral irritant zingerone: Relationship to frequency of chili consumption. Physiology \& Behavior, 60, 617-624.

Pringle, J. W. S., \& WiLson, V. J. (1952). The response of a sense organ to a harmonic stimulus. Journal of Experimental Biology, 29, 220-234.

Rodieck, R. W. (1973). The vertebrate retina: Principles of structure and function. Oxford: Freeman.

SCHARF, B. (1983). Loudness adaptation. In J. V. Tobias \& E. D. Schubert (Eds.), Hearing research and theory (Vol. 2, pp. 1-56). New York: Academic Press.

SchARF, B. (1991). Loudness adaptation measured by the method of successive magnitude estimation. In S. J. Bolanowski \& G. A. Gescheider (Eds.), Ratio scaling of psychological magnitudes (pp. 199214). Hillsdale, NJ: Erlbaum.

Segall, J. E., Block, S. M., \& Berg, H. C. (1986). Temporal comparisons in bacterial chemotaxis. Proceedings of the National Academy of Sciences, 83, 8987-8991.

Shapley, R., \& Enroth-Cugell, C. (1984). Visual adaptation and retinal gain controls. In N. N. Osborne \& G. J. Chader (Eds.), Progress in retinal research (3rd ed., pp. 263-346). Oxford: Pergamon.

Solomon, R. L. (1977). An opponent-process theory of acquired motivation: The affective dynamics of addiction. In J. D. Maser \& M. E. P. Seligman (Eds.), Psychopathology: Experimental models (pp. 67103). San Fransisco: W. H. Freeman.
Solomon, R. L., \& CoRBiT, J. D. (1974). An opponent-process theory of motivation: I. Temporal dynamics of affect. Psychological Review, 81, 119-145.

Stevens, J. C., \& Stevens, S. S. (1963). Brightness function: Effects of adaptation. Journal of the Optical Society of America, 53, 375-385.

Stevens, S. S. (1951). Handbook of experimental psychology. New York: Wiley.

Teghtsoonian, R. (1973). Range effects in psychophysical scaling and a revision of Stevens' law. American Journal of Psychology, 86, 3-27.

Tooby, J., \& Cosmides, L. (1992). The psychological foundations of culture. In J. H. Barkow, L. Cosmides, \& J. Tooby (Eds.), The adapted mind: Evolutionary psychology and the generation of culture (pp. 19136). New York: Oxford University Press.

VERRILlo, R. T. (1985). Psychophysics of vibrotactile sensation. Journal of the Acoustical Society of America, 77, 225-232.

Viemeister, N. F. (1979). Temporal modulation transfer functions based upon modulation thresholds. Journal of the Acoustical Society of America, 66, 1364-1380.

Wilson, V. J., \& Melvill Jones, G. (1979). Mammalian vestibular physiology. New York: Plenum.

\section{NOTE}

1. Because we are applying the term adaptation broadly in this article, we will be describing the behavior of receptors, neurons, sensory systems, and whole organisms at various times. We will generally not distinguish among them, unless noted otherwise.

(Manuscript received October 20, 2008; revision accepted for publication June 29, 2009.) 